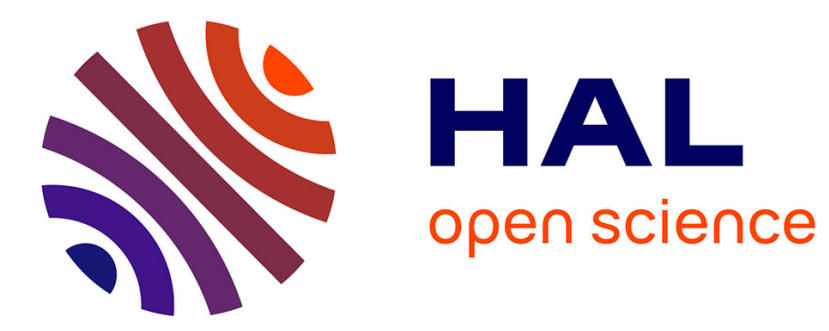

\title{
Une lettre d'époque classique à Thasos
}

Natacha Trippé

\section{To cite this version:}

Natacha Trippé. Une lettre d'époque classique à Thasos. Bulletin de Correspondance Hellenique, 2015, 139-140.1, pp.43-65. hal-01738154

\section{HAL Id: hal-01738154 https://hal.science/hal-01738154}

Submitted on 9 Dec 2019

HAL is a multi-disciplinary open access archive for the deposit and dissemination of scientific research documents, whether they are published or not. The documents may come from teaching and research institutions in France or abroad, or from public or private research centers.
L'archive ouverte pluridisciplinaire HAL, est destinée au dépôt et à la diffusion de documents scientifiques de niveau recherche, publiés ou non, émanant des établissements d'enseignement et de recherche français ou étrangers, des laboratoires publics ou privés. 


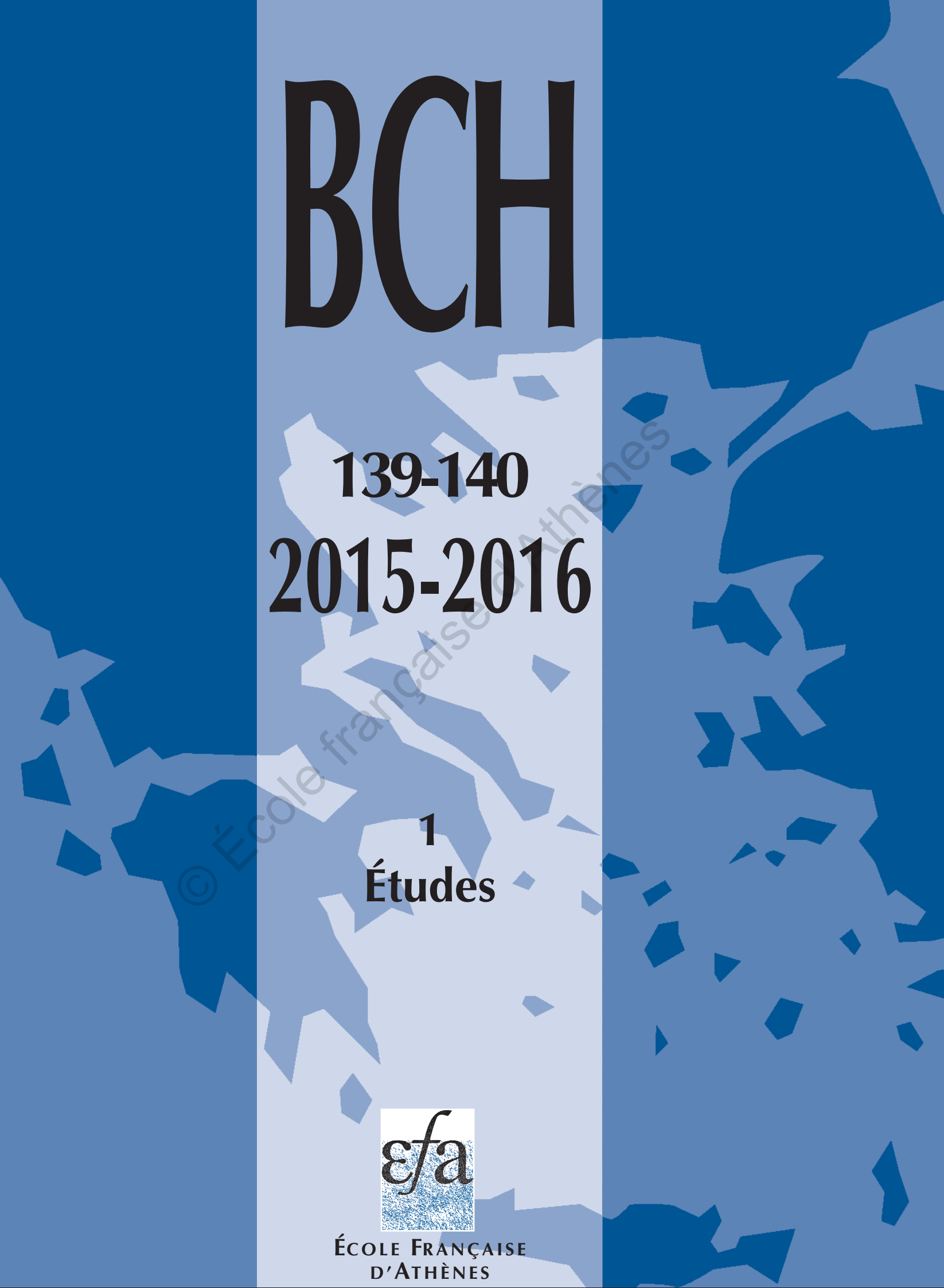

B ULLET IN DE CORRESPON D NCE HELLÉN IQUE 


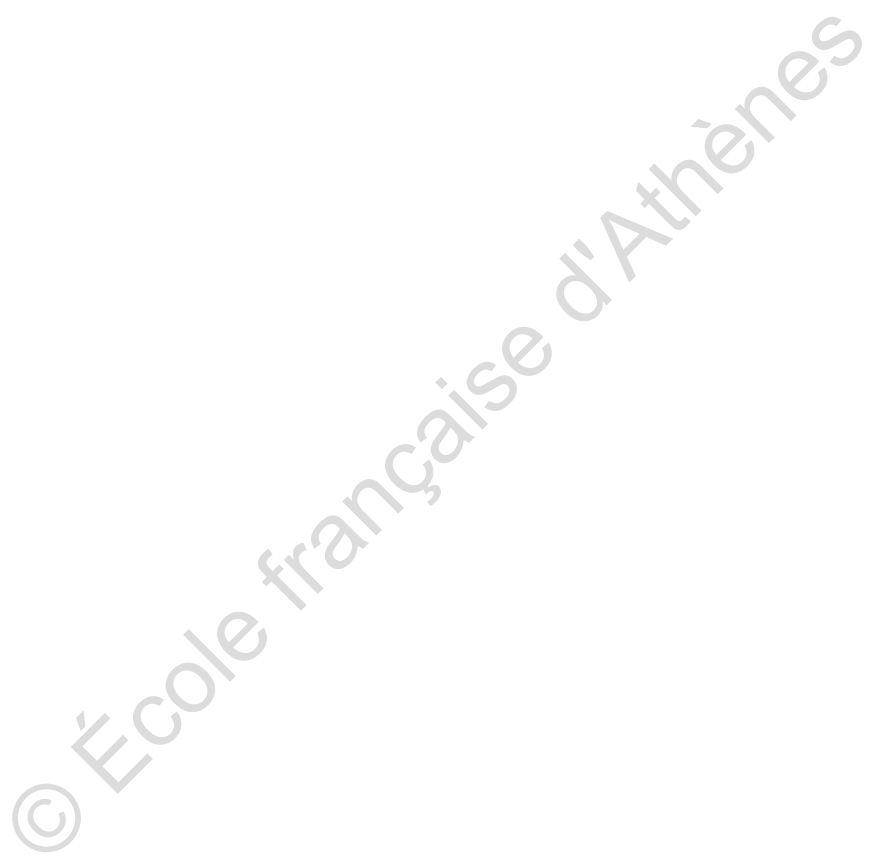




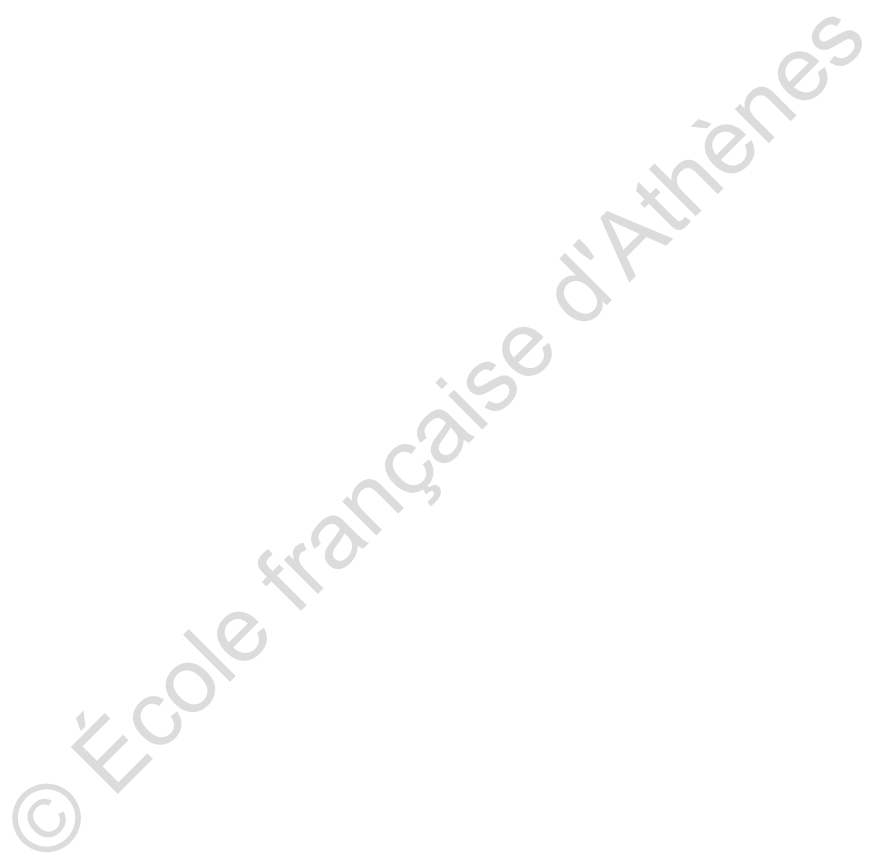


É C O L E F R A N ÇA I S E D'A T H È N E S

\author{
B U L L E T I N \\ DE CORRESPONDANCE \\ HELLÉNI QUE
}

$\mathrm{BCH}$

$139-140$

2015-2016 


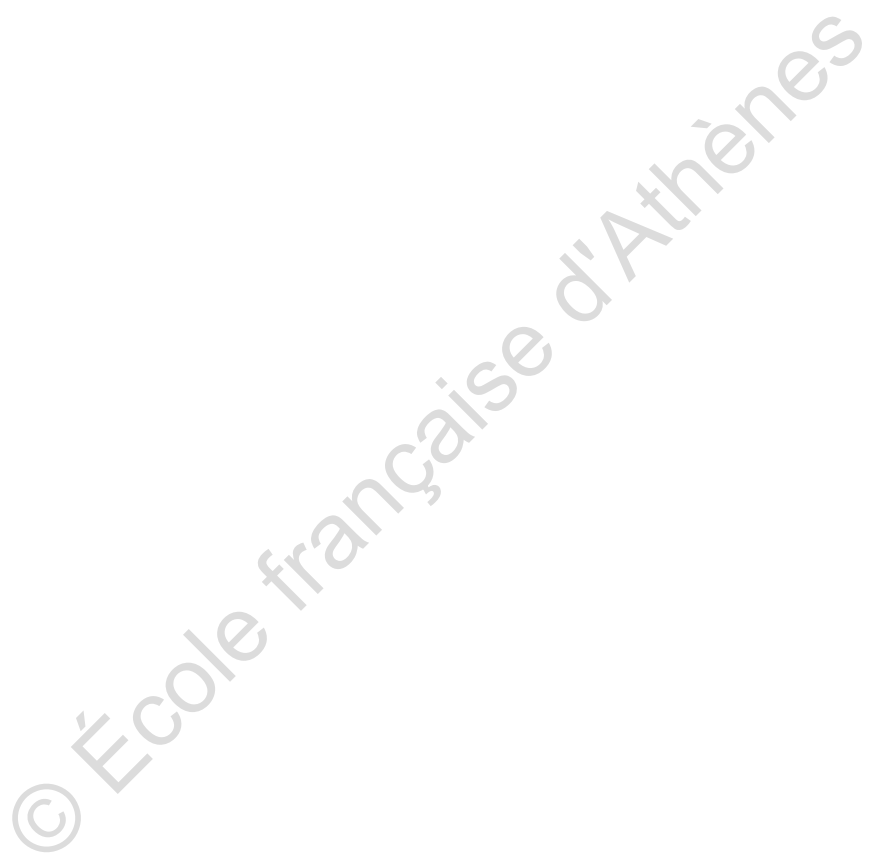


É C O L E F R A N ÇA I S E D'A T H È NE S

\author{
B U L L E T I N \\ DE CORRESPONDANCE \\ HELLÉNIQUE
}

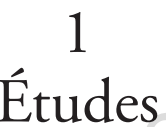

$\mathrm{BCH}$

$139-140$

2015-2016 


\title{
É C O L E F R A N Ç A I S E D'A T H È N E S
}

\author{
B U L L E T I N \\ DE CORRESPONDANCE \\ HELLÉNIQUE \\ $139-140.12015-2016$
}

Comité de rédaction : Alexandre Farnoux, directeur Géraldine HuE, responsable des publications

\section{COMITÉ DE LECTURE}

Le comité de lecture de l'École française d'Athènes est composé de trois membres de droit et de neuf membres désignés par le conseil scientifique sur proposition du directeur. Sa composition actuelle est la suivante (conseil scientifique de l'École française d'Athènes du 25 juin 2012) :
Membres
- le directeur de l'École française d'Athènes : Alexandre Farnoux
de droit
- le directeur des études antiques et byzantines : Julien Fournier
- le directeur des études modernes et contemporaines : Anastassios ANASTASsiadis
Membres $\quad$ Sont membres désignés des personnalités scientifiques françaises ou étrangères (mais francophones), reconnues et désignés de dimension internationale. Le choix en est fait de manière à assurer la meilleure représentation possible des champs disciplinaires concernés. Leur mandat coïncide avec la durée d'un contrat quinquennal.
- Polixeni Adam-Veleni, Directrice du musée archéologique de Thessalonique
- Olivier Deslondes, Professeur des Universités, Université Lyon 2-Lumière
- Emanuele Greco, Directeur de l'École italienne d'Athènes
- Jean Guilaine, Professeur au Collège de France
- Miltiade B. Hatzopoulos, Directeur de recherche, Directeur du Centre de recherche sur l'Antiquité gréco-romaine (Fondation nationale de la recherche [EIE] - Athènes)
- Catherine Morgan, Directrice de l'École britannique d'Athènes
- Kosmas Pavlopoulos, Professeur à l'Université Harokopio d'Athènes
- Jean-Pierre Sodini, Professeur émérite de l'université Paris 1 - Panthéon-Sorbonne
- Georges Tolias, Directeur de recherche en histoire contemporaine, Institut de recherche néo- hellénique (Fondation nationale de la recherche [EIE] - Athènes)

Le comité de lecture fait appel en tant que de besoin à des experts extérieurs.

Révision des textes : EFA, Sophie Duthion

Traduction et révision des résumés en grec : Pavlos Karvonis

Traduction et révision des résumés en anglais : Katie Low

Réalisation en PAO : EFA, Guillaume Fuchs

Impression et reliure : n.v. PEETERS s.a.

(C) École française d'Athènes, 2016

6, rue Didotou GR - 10680 Athènes www.efa.gr

Dépositaire : de Boccard Édition-Diffusion 11, rue de Médicis F - 75006 Paris www. deboccard.com

ISBN 978-2-86958-286-6

ISSN 0007-4217

Reproduction et traduction, même partielles, interdites sans l'autorisation de l'éditeur pour tous pays, y compris les États-Unis. 


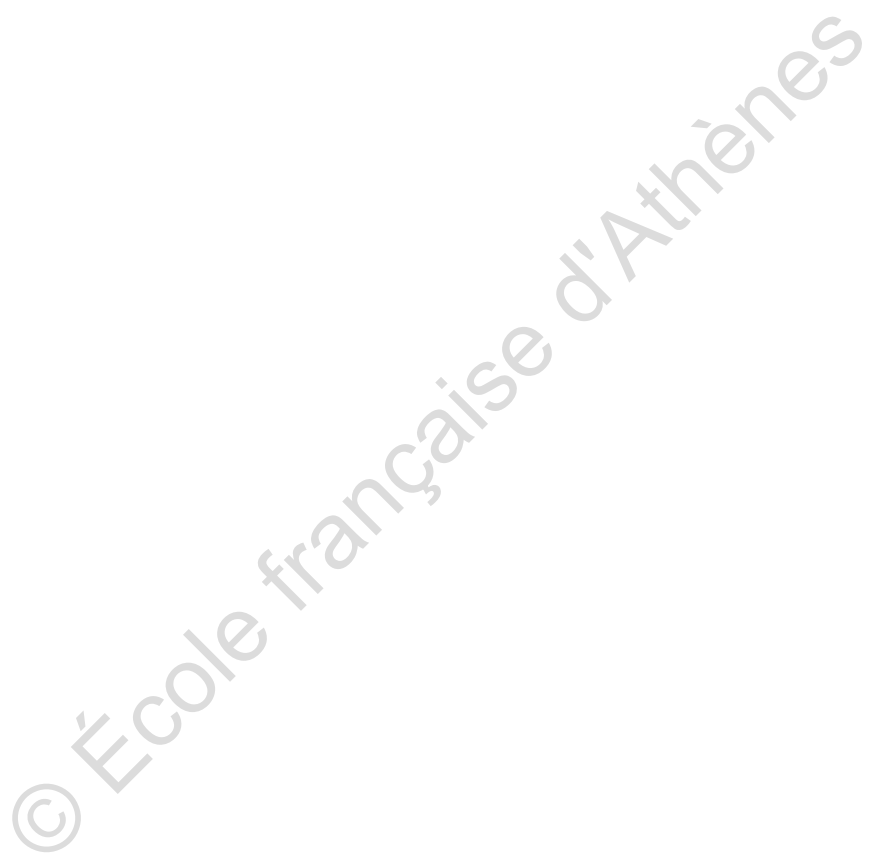




\section{AVIS AUX LECTEURS}

\section{Chronique en ligne}

Partageant une longue tradition, l'École française d'Athènes et la British School at Athens diffusent auprès de la communauté scientifique le résultat de l'activité archéologique conduite en Grèce et dans certaines régions du monde hellénique. Depuis 1920, l'École française d'Athènes consacre une partie du Bulletin de correspondance hellénique à la chronique des travaux archéologiques réalisés en Grèce, à Chypre et, selon un rythme bisannuel, dans le Bosphore cimmérien. De son côté, la British School at Athens compile un bilan annuel similaire, Archaeology in Greece, publié en association avec la Society for the Promotion of Hellenic Studies comme partie constitutive des Archaeological Reports depuis 1955. Chacune des deux institutions avait un double défi à relever : faire face à une documentation croissante, d'une part; utiliser des outils plus performants pour mieux faire circuler l'information scientifique et en permettre une meilleure utilisation, d'autre part. — L'École britannique a accepté sans hésitation le projet d'un programme commun que lui a proposé l'École française d'Athènes et les deux institutions ont décidé d'unir leurs efforts, pour proposer depuis la fin de l'année 2009 une Chronique des fouilles en ligne consultable sur http://chronique.efa.gr.

Outre les articles relatifs à des opérations de terrain ou relevant de l'archéométrie, le second fascicule du $B C H$ ne comprend donc plus désormais que les " Rapports sur les travaux de l'École française d'Athènes " proposés par les responsables de missions ou de programmes.

\section{AVIS AUX AUTEURS}

Depuis la parution du $B C H 130$ (2006), les tirages à part sont fournis aux auteurs sous format électronique et sont uniquement destinés à une utilisation privée. L'École française d'Athènes conserve le copyright sur les articles, qui ne peuvent donc être mis en accès libre sur quelque base de données ou par quelque portail que ce soit. — L'ensemble de la livraison sera disponible sur le portail Persée trois ans après sa parution (www.persee.fr). 


\section{SOMMAIRE DE LA LIVRAISON}

Laetitia Phialon

Une place pour l'artisanat mycénien? Remarques sur les espaces de production

et d'activités artisanales à l'Helladique Récent.

Natacha TRIPPÉ

Une lettre d'époque classique à Thasos.

Patrice HАMоN

Etudes d'épigraphie thasienne, IV. Les magistrats thasiens du IVe s. av. J.-C.

et le royaume de Macédoine.

Athanasios Tziafalias, Laurence Darmezin

Dédicaces d'affranchis à Larissa (Thessalie).

Benedetto Bravo, Aleksander Wolicki

Un katadesmos du banquier Pasiôn (SEG LIII 256)

$211-236$

Nathan BADOud

Note sur trois inscriptions mentionnant des Rhodiens morts à la guerre. Contribution à

l'étude des relations entre Rhodes et Rome à la fin du IVe s. av. J.-C.

Rachel NoueT

La " déesse au serpent " de Délos et l'aménagement des Propylées déliens

à l'époque classique.

Frédéric Herbin, François QueYrel

Les monuments attalides du Dromos à Délos (I) : la "base des Galates"

Pavlos Karvonis, Jean-Jacques Malmary

Les mezzanines déliennes : étude technique et contexte archéologique. $321-344$

Nathan Badoud, Myriam Fincker, Jean-Charles Moretti

Les monuments érigés à Délos et à Athènes en l'honneur de Ménodôros, pancratiaste et lutteur. $345-416$

Alain Bouet, Enora Le QuÉRÉ

Les thermes impériaux de Délos : l'infrastructure publique d'une ville ö $\delta \eta \lambda \lambda_{\diamond}$ ? $417-462$

Ludovic THÉLY

Inscriptions d'Amathonte XI. Un autel en l'honneur de Ptolémée X

et Bérénice III découvert aux abords Sud-Ouest de l'agora 463-484 


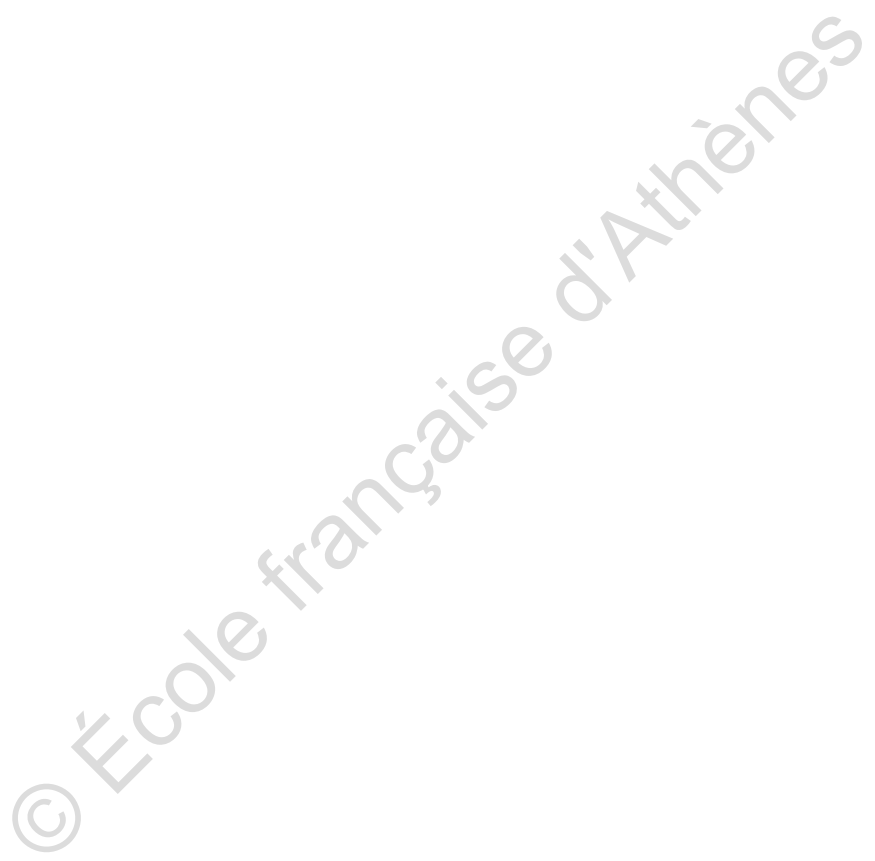




\title{
Une lettre d'époque classique à Thasos
}

\author{
Natacha TRIPPÉ
}

RÉsumÉ Cet article présente un document trouvé dans les fouilles de l'École française à Thasos en 1969. Daté de l'époque classique, ce texte fut inscrit sur une tablette avant cuisson, premier exemple de ce type de support sur ce site. Après une étude du texte et de ses caractéristiques paléographiques et linguistiques, on expose les éléments qui laissent penser que l'on a affaire aux premières lignes d'une lettre. En plus de présenter certaines graphies distinctes de celles de l'épigraphie lapidaire et des anthroponymes nouveaux, ce texte fournit une attestation supplémentaire du culte de Zeus Patrôos à Thasos.

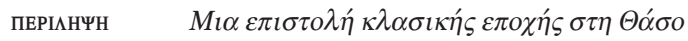

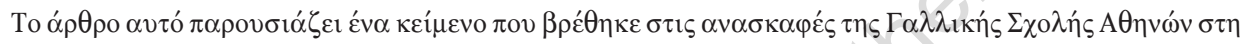

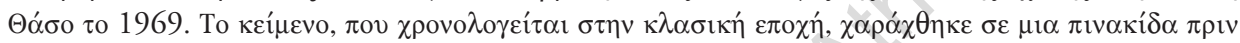

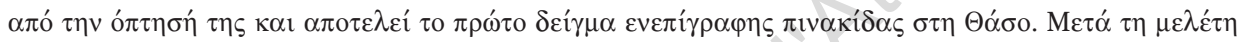

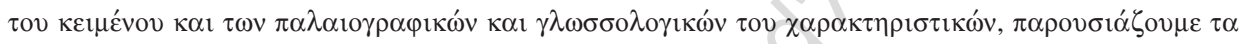

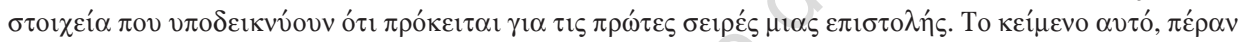

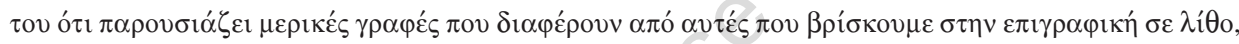

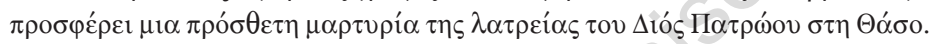

SUMmary Aletter from the Classical period found in Thasos

This article focuses on a document discovered in 1969 during excavations led by the French School at Athens in Thasos. Dated to the Classical period, the text was inscribed on a clay tablet before firing and represents the very first inscription from the site written on this type of support. After examining the paleographic and linguistic characteristics of the text, this article sets out some elements which suggest that it may be the first few lines of a letter. In addition to containing some written forms different from those found in lapidary epigraphy and some new personal names, this text provides further testimony for the worship of Zeus Patroos in Thasos. 


\section{À PROPOS DE L'AUTEUR}

N. TrIPPÉ, université Bordeaux-Montaigne, Ausonius UMR 5607.

\section{NOTE LIMINAIRE}

Je tiens à témoigner ici ma reconnaissance à M. O. Picard qui m’a confié la publication de ce document. J'adresse mes sincères remerciements à $\mathrm{M}^{\mathrm{me}} \mathrm{D}$. Malamidou, directrice du Musée de Thasos, qui me permet de travailler au musée dans d'excellentes conditions. Je remercie également M.-Fr. Billot qui a bien voulu me communiquer ses observations sur les terres cuites architecturales trouvées dans cette fouille, ainsi que $\mathrm{Cl}$. Prêtre et $\mathrm{Ch}$. Kritzas pour leurs observations sur le texte inscrit. Mes remerciements s'adressent aussi à Ph. Collet qui a réalisé les photographies du document, Fl. Duval qui en a effectué un moulage, et G. Biard qui m’a aidée dans la réalisation technique du fac-similé. Enfin, je tiens à remercier vivement S. Minon et P. Hamon pour leurs relectures attentives et leurs conseils avisés.

\section{ABRÉVIATIONS BIBLIOGRAPHIQUES}

Ceccarelli 2013 = P. Ceccarelli, Ancient Greek Letter Writing. A Cultural History (600-150 BC).

Pouilloux 1954 = J. Pouilloux, Recherches sur l'histoire et les cultes de Thasos, EtThas III.

TRIPPÉ 2012 = Écriture et alphabet dans les inscriptions archä̈ques thasiennes. Étude préliminaire, mémoire inédit de l'École française d'Athènes présenté à l'Académie des inscriptions et belles-lettres (2012). 
En 1969, lors de la fouille menée par O. Picard d'un terrain à Thasos dit terrain Apostolidis, est apparue, parmi le matériel mis au jour, une petite plaque de terre cuite portant une inscription de quelques lignes, de lecture difficile mais dont les éléments méritent d'être exposés.

\section{CONTEXTE ARCHÉOLOGIQUE}

\section{i. 1. Stratigraphie}

Fouillé à deux reprises en 1967 et 1969, le terrain Apostolidis se trouve sur les restes d'un quartier résidentiel remontant à l'époque archaïque, quartier situé non loin de la porte du Silène, à une centaine de mètres au Sud / Sud-Est de l'Héracleion et sur le bord NordEst de l'actuelle rue de l'École française' (fig. I).

La fouille menée par O. Picard a mis en évidence la stratigraphie suivante ${ }^{2}$ : sous un niveau romain du Bas-Empire, daté par des tessons gaufrés et des monnaies, apparaît le niveau hellénistique, constitué en certains endroits de remblais de sable assez épais ${ }^{3}$. Sous les niveaux hellénistiques se trouvent directement les niveaux archä̈ques qui étaient occupés par les vestiges de ce qui fut tout d'abord interprété comme une seule maison de dimensions imposantes.

Cette habitation était constituée d'une pièce centrale de $5,20 \mathrm{~m}$ sur $4,80 \mathrm{~m}$, comprenant en son centre un foyer. Deux autres pièces, à l'Est et à l'Ouest, furent repérées sans être dégagées. Cette pièce centrale est bordée au Nord par une vaste cour mais ne communique pas avec elle. La cour est elle-même bordée, sur toute la longueur de son côté Est, d'un portique ouvert à l'Ouest et faisant retour au Sud (fig. 2).

La cour n'ayant aucune relation avec la pièce centrale, le mur les séparant est par conséquent un mur mitoyen entre deux maisons, l'une située au Nord de ce mur et

1. Sur la fouille, voir la chronique des fouilles du $B C H$ ainsi que les carnets de fouilles et un rapport conservés à l'EFA (O. Picard, BCH 92 [1968], p. 1082-1094; O. Picard, Carnet de fouille, Ctha 106 1969; O. PicARD, $B C H 94$ [1970] p. 812-818).

2. Nous ne mentionnons ici que les informations essentielles dont nous disposons concernant la stratigraphie. Une reprise des données de la fouille ainsi qu'une étude approfondie de l'ensemble du mobilier feront l'objet d'une publication conjointe par M. Perron et N. Trippé. Notons d'emblée que dans ce matériel se trouvent deux graffites inscrits sur des terres cuites architecturales qui seront en outre publiés avec les autres graffites thasiens dans le cadre de la préparation du corpus épigraphique actuellement en cours.

3. Ces remblais contenaient de très nombreux tessons archaïques, des monnaies de bronze et quelques tessons à figure rouge. En certains endroits sont apparus deux remblais superposés distingués par les fouilleurs en couches $3 a$ et $3 b$. 


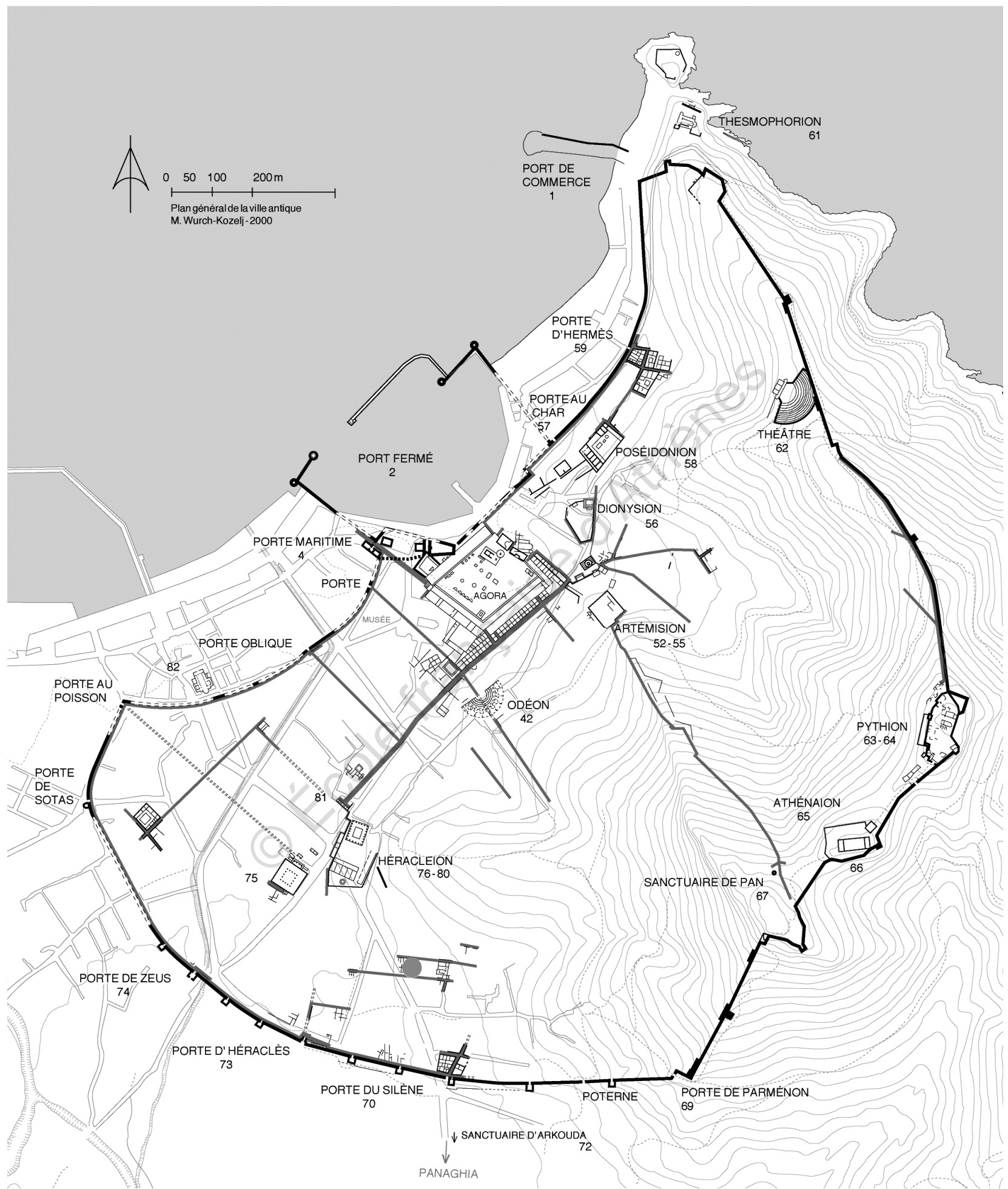

Fig. I — Plan de situation (d'après Y. Grandjean et Fr. Salviat, Guide de Thasos ${ }^{2}$ [2000], fig. 12). 


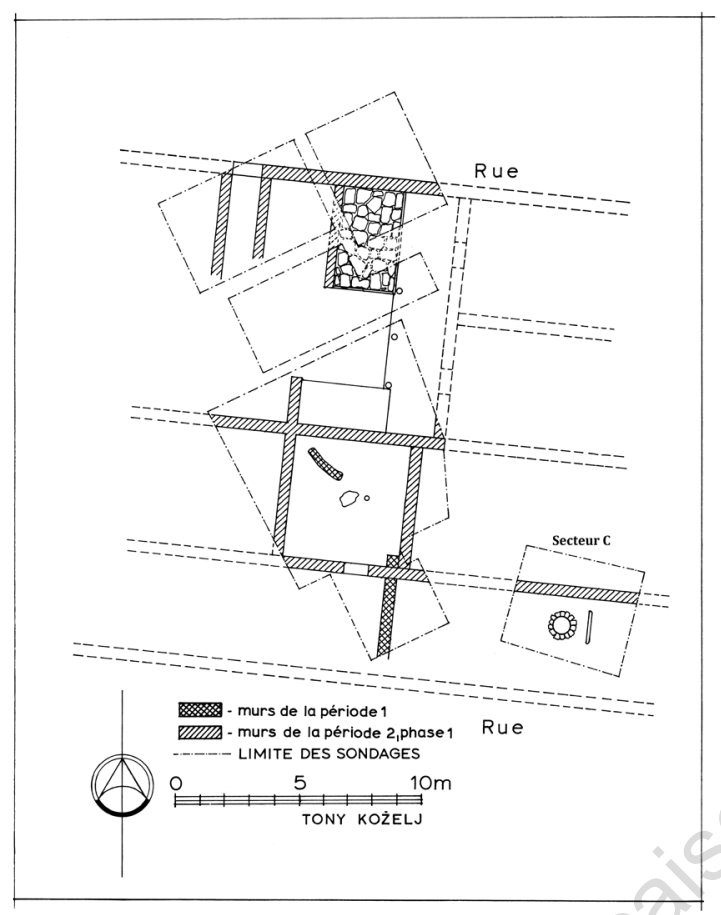

Fig. 2 - Plan de l'implantation des sondages à l'issue de la fouille.

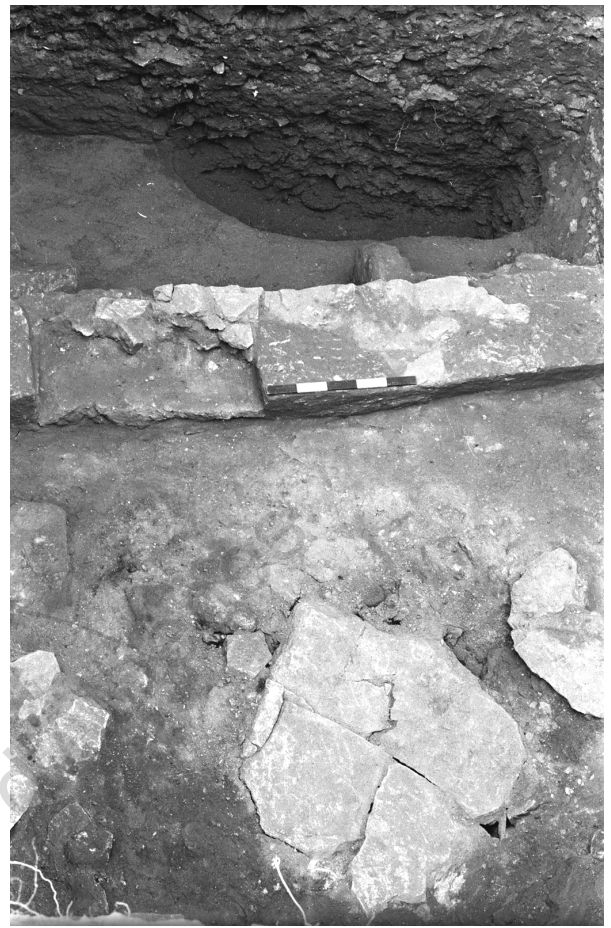

Fig. 3 - Sondage C : au centre le mur Est-Ouest, la fosse au Nord, le puits scellé au premier plan. Vue depuis le Sud (cl. EFA).

comportant une cour; l'autre au Sud de ce mur et constituée de cette pièce centrale. On a donc affaire ici à un îlot d'habitation, comprenant au moins deux maisons ${ }^{4}$.

La fouille fut par ailleurs élargie par l'ouverture d'un nouveau secteur situé au Sud-Est de la zone, sur le terrain Divanakis (secteur C) ${ }^{5}$. Sous les niveaux tardifs, ce sondage a permis de retrouver les niveaux les plus anciens et de mettre en évidence le prolongement du mur Sud du bâtiment archaïque, visible sur toute la longueur du sondage et détruit jusqu'au niveau des fondations. La majeure partie du sondage était remplie d'un remblai constitué de sable mêlé de gneiss, passant au-dessus de l'arasement

4. Cette interprétation est due à Y. Grandjean (Y. Grandjean, Recherches sur l'habitat thasien à l'époque grecque, EtThas XII [1988] p. 340-343 et 441-444).

5. Un sondage de $5 \mathrm{~m}$ sur $4 \mathrm{~m}$ y fut implanté. La fouille a d'abord rencontré plusieurs niveaux de sable dans lesquels ne se trouvait d'autre structure qu'un égout tardif orienté Sud-Nord fait de petits blocs de gneiss et de marbre, sans couverture ni dallage. 
du mur, très riche en tessons. Au Sud du mur du bâtiment archaïque, c'est-à-dire à l'extérieur du bâtiment, est apparu sous le remblai un dallage de gneiss ainsi qu'un puits, scellé par une grande dalle de gneiss et demeuré intact depuis son abandon, très pauvre en matériel ${ }^{6}$.

Enfin, entre le mur et la paroi Nord du sondage, creusée dans une couche de sable jaune brillant, très indurée et vierge de matériel, est apparue une fosse remplie d'un sable de gneiss jaune sombre, contenant de nombreux tessons. C'est dans cette fosse qu'a été trouvé notre document (fig. 3 ).

\section{2. Datations}

La fouille des niveaux archaïques du terrain Apostolidis a ainsi mis au jour un large édifice, présentant deux phases d'occupation : une première phase, illustrée par la couche dite $5 \mathrm{c}$, et une seconde phase, $5 \mathrm{a}$, toutes deux séparées par une couche de destruction liée à un incendie (5b). Dans son second état, le bâtiment ne fut que partiellement reconstruit. Après sa destruction, le portique au Nord qui appartient à la première phase d'occupation (5c) fut ainsi recouvert par une couche de sable tandis que la cour demeura à ciel ouvert. De même, le remblai révélé dans la fouille du secteur $\mathrm{C}$ correspond au réaménagement de cette zone qui fut laissée libre de construction.

L'étude de la céramique ${ }^{7}$ a permis de proposer des premiers éléments de datation : le premier niveau d'occupation est à dater, d'après les tessons orientalisants, de la fin du viIe/ début du vi ${ }^{e}$ s. av. J.-C. ${ }^{8}$. La couche de sable de gneiss 5 a correspondant au remblaiement en vue du réaménagement partiel de l'édifice comportait quant à elle beaucoup de matériel dont les éléments les plus récents indiquent que la reconstruction se situerait assez tard dans le vie $\mathrm{s}$. : selon les premières observations d'A. Coulié, la céramique attique indique ainsi un terminus post quem vers $525^{\circ}$.

6. Un seul tesson permet de proposer une datation : il s'agit d'un tesson clazoménien datant du dernier quart du vies.

7. Seules certaines catégories de mobilier ont été étudiées, comme les terres cuites architecturales par M.-Fr. Billot, ou la céramique thasienne à figures noires par A. Coulié (A. Coulié, La céramique thasienne à figures noires, ÉtThas XIX [2002], p. 220). L'étude prévue de l'ensemble de ce matériel sera par conséquent susceptible d'affiner certaines de ces datations.

8. Ce matériel fut retrouvé dans les tranchées de fondation et sur le sol (O. PiCARD [n. 1], A. Coulié [n. 7]).

9. Notons que ces niveaux archaïques ont succédé à un niveau d'occupation plus ancien, visible seulement en certains endroits et illustré par le mur en arc de cercle au centre de la pièce centrale et un mur au Sud (structures relevant de la période dite "période 1 » sur la fig. 2), qui daterait de la seconde moitié du viI $s$. 


\section{3. INTERPRÉTATION}

Les données de la fouille confirment la fonction domestique de l'édifice ${ }^{10}$. Cet habitat archaïque, dont l'état le plus ancien remonterait au viI ${ }^{\mathrm{e}}$ s., est donc composé d'au moins deux maisons séparées par le mur mitoyen Est-Ouest mis en évidence dans la pièce dite centrale. La maison au Nord, dont les fouilleurs ont mis au jour la cour dans ses deux états, ouvrirait sur la rue qui la borde au Nord, d'orientation Est-Ouest et descendant de la colline, tandis que la maison au Sud ouvrirait sur une cour dont les vestiges sont visibles à la fouille du sondage C : en effet, selon Y. Grandjean, le puits et le dallage grossier mis au jour dans ce sondage sont les vestiges d'une cour aux dimensions allongées qui bordait la maison ouvrant au Sud et en distribuait plusieurs pièces ${ }^{11}$.

En ce qui concerne la fosse du sondage $\mathrm{C}$ qui nous intéresse plus particulièrement ici, la stratigraphie semble indiquer qu'elle fut creusée dans le niveau d'occupation 5c. Du contenu de cette fosse, seules les terres cuites architecturales ont fait l'objet d'un diagnostic complet. Le mobilier présente ainsi un faciès s'étendant sur une longue durée, allant de la seconde moitié du $\mathrm{VI}^{\mathrm{e}} \mathrm{s}$. pour les plus anciennes ${ }^{12}$, jusqu'à l'époque tardoclassique/hellénistique pour les plus récentes ${ }^{13}$. Ce matériel, qui comporte des terres cuites architecturales appartenant au deuxième état du bâtiment archaïque mais aussi au bâtiment tardo-classique, fournit donc un terminus ante quem pour le remplissage de cette fosse à la fin de l'époque classique ou au début de l'époque hellénistique ${ }^{14}$. Il est possible que le mobilier appartenant aux édifices antérieurs, dans lequel se trouve le présent document, ait été enfoui à l'occasion d'un nouveau remaniement réalisé à cette époque ${ }^{15}$.

\section{PRÉSENTATION DU DOCUMENT}

Plaque de terre cuite ${ }^{16}$ brisée à gauche et dans sa partie inférieure (fig. 4-5). Bien qu'irrégulier, le bord gauche subsiste dans sa partie supérieure sur une hauteur de $2,1 \mathrm{~cm}$. Le bord droit est intact. La face arrière est lisse.

10. Ce que l'étude du matériel permettra de confirmer.

11. Y. Grandjean (n. 4), p. 340-343, p. 441-444 et planches.

12. Il s'agit des terres cuites portant les inventaires 7977/7917 et 7913. Selon M.-Fr. Billot, ces deux antéfixes appartiendraient à l'édifice dans son deuxième état, après sa reconstruction.

13. Il s’agit de tuiles à méandre en relief que M.-Fr. Billot attribue à un état tardo-classique/hellénistique du toit du deuxième édifice.

14. L'étude d'ensemble de la céramique permettra là encore de préciser cette datation.

15. Ce remaniement pourrait alors correspondre à la mise en place du remblai hellénistique trouvé ailleurs dans le bâtiment (noté niveau 3 par les archéologues).

16. La pâte présente de grosses inclusions de mica typiques, selon M. Perron, de la céramique du Nord de l'Égée (M. Perron, La production et la diffusion des céramiques de style à bandes à Argilos et dans le nord de 
La plaque fut retrouvée en 1969 par O. Picard dans les fouilles du terrain Apostolidis, dans le secteur C (sondage Divanakis).

Inscription sur 9 lignes. La coupe des lignes respecte la coupe des mots.

Inv. П 5196. H. max. cons. : 5,8 cm; 1. : 9,5 cm; ép. : $1,8 \mathrm{~cm}$. H. 1. : 0,5 cm. Phot. (inédites, N562-1 à 5 et fig. 4 : N607-24,25). Collation. Document inédit.

Plusieurs aspects rendent la lecture du document difficile. La technique de gravure, tout d'abord : le texte a été inscrit sur l'argile crue, de sorte que l'enfoncement de la pointe du stylet a laissé des débords. La disposition des lignes montant vers le haut et l'aspect inachevé de certaines lettres peuvent dénoter une écriture rapide ou à main levée.

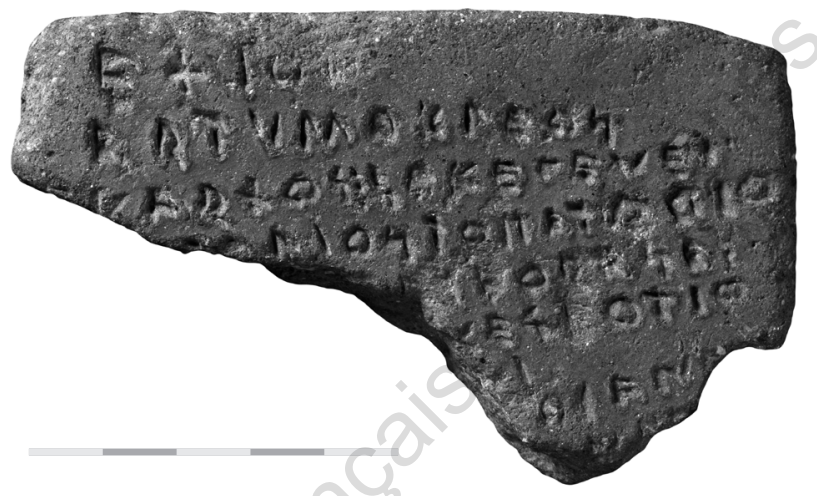

Fig. 4 - Fragment de plaque de terre cuite, musée de Thasos, inv. П 5196 (cl. EFA, Ph. Collet).

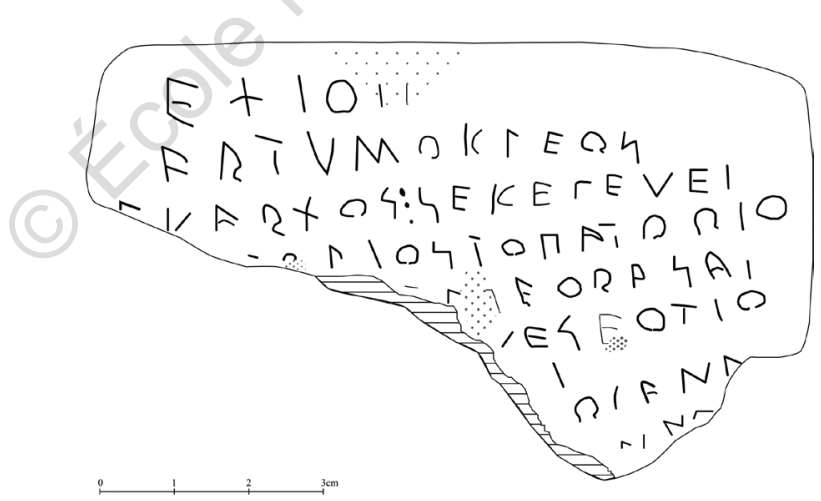

Fig. 5 - Fragment de plaque de terre cuite, fac-similé (N. Trippé).

l'Égée aux périodes archaïques et classiques, thèse, universités de Montréal et Paris I [2013]. La couleur claire de l'argile indique qu'elle fut cuite en milieu oxydant, dans le cadre d'une cuisson maîtrisée (voir ci-après). 
Une autre difficulté vient du fait qu'il est presque impossible de faire la distinction entre les $\Omega$ et les $\mathrm{O}$. Cela a une incidence directe sur la caractérisation de l'alphabet (voir cidessous).

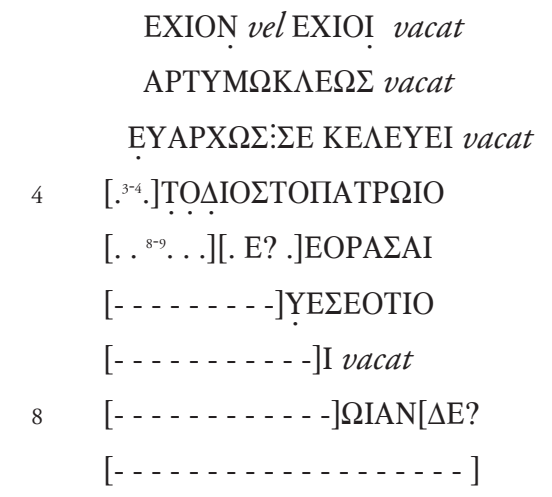

EXION vel EXIOI vacat

YM $\Omega \mathrm{K} \Lambda \mathrm{E} \Omega \Sigma$ vacat

EYAPX $\Omega \Sigma: 2$ E KE $\Lambda$ EYEI vacat

.

[.. . . .

[- - - - - Y YELEOTIO

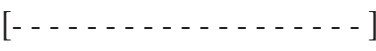

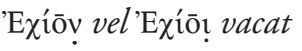

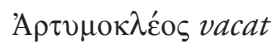

Ev̋ $\alpha \rho \chi \circ \varsigma$ : $\sigma \varepsilon \kappa \varepsilon \lambda \varepsilon v ́ \varepsilon \imath$ vacat

4

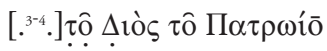

$\left[.{ }^{8-9} \ldots\right]\left[. \varepsilon ?\right.$. . ] $\varepsilon_{\varepsilon} \rho \alpha \sigma \alpha \iota$

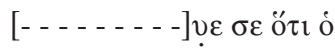

[- - - - - - - - ]I vacat

8

[- . . . . . - . . . . . . ]

Notes critiques :

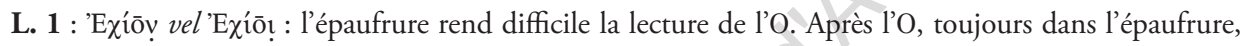
on distingue une haste verticale et peut-être une seconde haste dont on ne verrait que l'empreinte. Cette lettre n'est suivie d'aucune autre. L. 2 : la lecture de l'A, comme dans l'ensemble du document, fait difficulté : on distingue une haste verticale et deux hastes obliques peut-être reliées entre elles par une haste

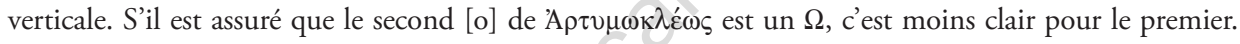
L. 3 : à gauche, une haste oblique et départ d'une haste verticale formant un angle. Puis on voit la haste verticale et une haste oblique prenant son départ dans la partie inférieure de la haste verticale de la lettre, ce qui invite à lire un Y. L. 4 : à gauche, on distingue dans la cassure la haste horizontale du T, légèrement oblique. La lettre qui suit est une lettre ronde, vraisemblablement un $\mathrm{O}$. La haste horizontale du $\Delta$ n'est pas apparente. L. 5 : de la première lettre on distingue une haste horizontale supérieure. Je distingue ensuite la partie supérieure d'une lettre angulaire $(\Lambda, E$ ?). Ensuite l'épaufrure ne laisse pas apparaître la lettre concernée. Je crois néanmoins distinguer une haste verticale avec un départ de haste horizontale vers la gauche (seconde moitié d'un П ou un T vite réalisé?). L. $\mathbf{6}$ : de la première lettre je distingue un morceau de haste oblique. Ce qui peut sembler une haste oblique dans la partie inférieure (qui ferait songer à un $\mathrm{X}$ en croix) est en réalité une entaille sur la surface de la plaque. Il peut ainsi s'agir d'un $\mathrm{N}$ ou d'un Y. Le tracé de la troisième lettre pose de réelles difficultés. La lecture sur le document montre un premier quart supérieur arrondi vers la gauche, suivi d'une haste horizontale vers la droite qui se prolonge par une haste verticale, de sorte que cela semble être un sigma possédant une haste verticale très arrondie, observation que confirme de manière assurée le moulage. La dernière lettre, qui semble être un $\mathrm{O}$, est toutefois plus petite et écrasée que les autres et légèrement suspendue. L. 7 : une haste verticale que j'interprète comme un I à moins qu'il ne s'agisse de la haste verticale d'un N. Malheureusement, l'usure du bord empêche de distinguer la lettre qui la précédait immédiatement. L. 8 : comme à la ligne au-dessus, la lettre précédant immédiatement l' $\Omega$ a disparu. Pour la dernière lettre, l'angle supérieur d'une lettre angulaire apparaît, peut-être un $\Delta$. L. 9 : n'apparaissent que les parties supérieures de lettres angulaires (N, $\Delta, \mathrm{E}$ ?). 


\section{1. RemARQUes SUR LA PALÉOgRAPHIE}

Le caractère privé du document conjugué à la nature du support implique qu'il est assez malaisé de se fonder sur les documents de l'épigraphie lapidaire ou sur les documents à caractère officiel pour élaborer une analyse de la forme des lettres. En outre, l'écriture des graffites et, en règle générale, des documents privés ne suit pas la même évolution que les documents à caractère officiel. En ayant à l'esprit ces réserves, on peut toutefois avancer certains éléments de comparaison à travers quelques ductus caractéristiques (tabl. I ${ }^{17}$.

Dans l'ensemble du document, le tracé des alpha montre une certaine négligence, ou du moins un certain empressement. L'A est ainsi constitué de deux hastes obliques et d'une haste médiane qui prend son départ dans la première mais ne touche pas réellement la seconde haste oblique $(1.1,3)$, de sorte qu'on aboutit à une sorte d'alpha " ouvert à droite ". Ces A s'apparentent toutefois aux A archaïques, isolés dans l'épigraphie thasienne en type $1(A){ }^{18}$. Dans ce cas précis, le fait d'écrire dans l'argile crue conduit à des formes de lettres propres au type de support.

Les epsilon de ce document sont particulièrement soignés : ils se composent d'une haste verticale penchée dans le sens de l'écriture et de hastes horizontales toutes de même longueur ${ }^{19}$.

Le lambda est conforme au $\Lambda$ des autres alphabets cycladiques, présentant une haste verticale et une haste oblique plus petite ${ }^{20}$. Bien que le document n'en présente qu'une occurrence, il faut noter qu'ici le lambda n'est pas celui caractéristique de l'alphabet parien $\Gamma$ qui fait son apparition dans les inscriptions thasiennes entre 500 et 470 (type 2).

Le M présente ici un angle interne, formé par les deux hastes obliques, qui descend jusqu'au niveau de la ligne d'écriture, ductus attesté à Thasos dès les premières inscriptions (type 1) jusqu'au début du ve s. où il ne descend plus que jusqu'au premier tiers de la lettre (type 2).

Le document ne présente qu'une occurrence du $n u$ (1. 8). La seconde haste verticale est légèrement penchée vers la droite, tandis que l'angle interne touche la ligne d'écriture.

17. Cette analyse se fonde sur le travail en cours mené sur l'étude de l'évolution de l'écriture dans les inscriptions thasiennes archaïques. Voir Trippé 2012, notamment le tableau des ductus, annexe 1 p. 97-99, dont nous présentons une partie infra (tabl. I).

18. Trippé 2012, p. 12. Ce type disparaît dans les premières années du ve s. où l'A adopte une forme plus droite.

19. La comparaison avec l'épigraphie lapidaire est dans ce cas peu opérante : l'E y est très nettement penché vers la droite jusque vers la fin du vi ${ }^{\mathrm{e}} \mathrm{s}$. (type 1) avant d'aborder une forme bien droite et quadrangulaire dans les documents du début du ve s. (type 3). Mais ici le fait qu'il soit penché s'explique par l'inclinaison générale de l'écriture de ce document. Il s'agit d'epsilon de fin de type 1.

20. Isolé en type 1 dans TRIPPÉ 2012. 


\begin{tabular}{|c|c|c|c|c|}
\hline$\varepsilon$ & $E \quad t$ & \begin{tabular}{ll|}
$E$ & \\
& $\mathrm{t} 2$
\end{tabular} & \begin{tabular}{ll|}
$E$ & \\
& t3 \\
\end{tabular} & \\
\hline К & $\begin{array}{ll}K_{\mathrm{t}} & \\
\mathrm{t} 1\end{array}$ & \begin{tabular}{ll|}
$\mathrm{K}$ & \\
& $\mathrm{t} 2$ \\
\end{tabular} & \begin{tabular}{ll|}
$\mathrm{K}$ & \\
& $\mathrm{t} 3$ \\
\end{tabular} & \\
\hline$\lambda$ & r $\mathrm{t} 1$ & \begin{tabular}{ll|}
$\Gamma$ & \\
& $\mathrm{t} 2$ \\
\end{tabular} & & \\
\hline $\boldsymbol{\mu}$ & $M{ }_{t 1}$ & $\begin{array}{ll}M & \\
& \mathrm{t} 2\end{array}$ & & \\
\hline $\mathbf{v}$ & $\begin{array}{ll}N & \\
& \mathrm{t} 1 \\
\end{array}$ & $\begin{array}{ll}N & \\
& \mathrm{t} 2 \\
\end{array}$ & \begin{tabular}{ll|}
$N$ & \\
& $\mathrm{t} 3$ \\
\end{tabular} & \\
\hline$\rho$ & $\begin{array}{ll}P & \\
& \mathrm{t} 1\end{array}$ & $\begin{array}{lll}R & \\
\end{array}$ & \begin{tabular}{ll|}
$R$ & \\
& $t 3$ \\
\end{tabular} & $\begin{array}{ll}P & \\
& t 4\end{array}$ \\
\hline $\boldsymbol{\sigma}$ & $\mathrm{t} 1$ & \begin{tabular}{ll|} 
& \\
& $\mathrm{t} 2$ \\
\end{tabular} & \begin{tabular}{ll|}
$S$ & \\
& $t 3$
\end{tabular} & \\
\hline v & $\begin{array}{ll}\mathrm{V} & \\
& \mathrm{t} 1\end{array}$ & $\begin{array}{lll}V & \\
& \mathrm{t} 2 \\
\end{array}$ & & \\
\hline$\chi$ & $\begin{array}{ll}\times \quad \mathrm{t} 1 \\
\end{array}$ & $\begin{array}{ll}+ \\
\mathrm{t} 2\end{array}$ & & \\
\hline
\end{tabular}

Tableau I - Tableau des lettres caractéristiques (d'après TRIPPÉ 2012).

Le rhô présente quant à lui un ductus bien attesté dans le reste de l'épigraphie archaïque thasienne: il est effectué en un seul tracé, la boucle formant un ovale descendant s'achevant par un petit appendice (type 2) qui deviendra, au fil de l'évolution, clairement indépendant (type 3) avant de disparaître (type 4$)^{21}$.

Enfin notons le type 1 de l'upsilon: deux hastes obliques dont l'angle rejoint la ligne d'écriture, sans support vertical, forme qui est en usage jusque vers 460 .

Si ces ductus sont ceux que l'on rencontre par ailleurs dans l'épigraphie thasienne, trois lettres méritent de retenir l'attention. Dans le travail mené sur l'écriture des inscriptions archaïques thasiennes, nous avons ainsi isolé, sous un type 3 , un seul exemple d'un $\mathrm{K}$ constitué d'une haste verticale et d'un demi-cercle tangent à celle-ci : il s'agit de l'inscription funéraire pour un certain Thrasyclès datée de la fin du vie ${ }^{2}{ }^{22}$. Notre document fournit donc un second exemple de ce tracé.

Les inscriptions thasiennes présentent un sigma à quatre branches qui adopte au $\mathrm{VI}^{\mathrm{e}} \mathrm{s}$. un tracé plus ou moins sinueux selon les documents. Au cours de la première moitié

21. Ce n'est qu'à la fin du vie s. que le tracé de la lettre présente une boucle nettement circulaire avec un appendice indépendant. Ce dernier disparaît aux alentours de 480-470 (Règlements cultuels du Passage des Théores [IG XII 8, 358] et Loi sur le vin et le vinaigre [Pouilloux 1954, n 7]).

22. Inv. 320. IG XII 8, 395 (photographie dans Pouilloux 1954, no 4). D'autres similitudes entre cette inscription et notre document sont par ailleurs remarquables : l'écriture est également penchée vers la droite, ce qui fait songer à une gravure rapide. S'y retrouvent aussi l'aspect général courbe des lettres ainsi que certains ductus spécifiques (P, E, Y). 
du ves., ce tracé devient plus angulaire, avec des hastes horizontales divergentes ${ }^{23}$. Mais notre document présente un sigma à trois branches (4) qui n'est pour le moment attesté qu'une seule fois dans l'épigraphie thasienne, dans une inscription funéraire datée des premières années $\mathrm{du} \mathrm{v}^{\mathrm{e}} \mathrm{s}$. $(500-480)^{24}$. Ce sigma à trois branches est également attesté à Naxos alors que, dans l'état actuel de nos connaissances, Paros utilise uniquement le sigma à quatre branches (type 2). Notre document constitue donc la seconde attestation de cette forme à Thasos ${ }^{25}$.

Enfin la forme du $\operatorname{chi}\left[\mathrm{k}^{\mathrm{h}}\right]$ dans ce texte est particulièrement remarquable. Les alphabets des Cyclades appartiennent, d'après la terminologie héritée de Kirchhoff, à la série des alphabets dits « bleus ", c'est-à-dire des alphabets orientaux, par opposition aux alphabets « rouges " occidentaux de Grèce centrale et occidentale ${ }^{26}$. Dans le système des alphabets dits bleus, l'occlusive vélaire aspirée $\left[\mathrm{k}^{\mathrm{h}}\right]$ a la forme $\mathrm{X}^{27}$. Aussi est-ce sans exception la forme attestée dans l'alphabet de Naxos et de Paros-Thasos ${ }^{28}$. Cette forme possède néanmoins une variante en croix droite $(+)$ attestée à une reprise à Paros ${ }^{29}$. Comme à Paros la forme la plus répandue pour $\left[\mathrm{k}^{\mathrm{h}}\right]$ à l'époque archaïque dans les inscriptions thasiennes est $\mathrm{X}^{30}$. On ne le rencontre sous forme de croix droite + que de manière sporadique : son attestation dans notre document est le troisième exemple de ce ductus à Thasos ${ }^{31}$.

23. La forme particulière du $\Sigma$ que présente la Loi sur le vin et le vinaigre (inv. 895, Pouilloux 1954, n 7), où les deux hastes horizontales divergentes sont reliées entre elles par deux hastes médianes qui forment en réalité une unique courbure, répond peut-être à l'aspect volontairement décoratif de l'écriture de cette inscription.

24. Inv. 318. Pouilloux 1954, no 5 (photographie dans Pouilloux 1954, pl. IV no 3 et N414-012). Nous avons isolé le sigma à trois branches en type 3 dans TRIPPÉ 2012.

25. Si l'on songe peut-être à une influence de Naxos sur le ductus de ce sigma à Thasos, une influence attique n'est évidemment pas à exclure et, peut-être même, à privilégier.

26. Ces dénominations trouvent leur origine dans la carte présentée en fin de volume par A. Kirchhoff, distinguant les différents alphabets par des couleurs, bleu, rouge, vert, selon la manière dont sont notées les lettres $\Phi, X, \Psi$ (A. KIRCHHOFF, Studien zur Geschichte des griechischen Alphabets ${ }^{4}$ [1887] et récemment R. D. Woodard, Greek Writing from Knossos to Homer. A linguistic Interpretation of the Origin of the Greek Alphabet and the Continuity of Ancient Greek Literacy [1997], p. 140). Le groupe des alphabets dits bleus se divise en "bleu foncé " et "bleu clair ". L'alphabet parien appartient à cette dernière catégorie.

27. Dans les alphabets occidentaux (rouges), [ $\left.\mathrm{k}^{\mathrm{h}}\right]$ a la forme $\downarrow$ et $[\mathrm{ks}]$ la forme X.

28. À Délos, les graffites sur les tessons retrouvés à l'Archegesion ont montré que les Déliens utilisaient un $\left[\mathrm{k}^{\mathrm{h}}\right]$ rouge contrairement à l'usage des îles voisines Naxos et Paros (Fr. Prost, "L'alphabet des Déliens à l'époque archaïque ", dans Chr. Müller, Fr. Prost (éds), Identités et cultures dans le monde méditerranéen antique, Mélanges Fr. Croissant [2002], p. 312).

29. IG XII 5, 216, fin vie s. Il s'agit de la dédicace d'une offrande de statue à Artémis par une certaine Télestodikè. Le + apparaît à la ligne 3 .

30. Isolé en type 1 dans TrIPPÉ 2012, tandis que + correspond au type 2 .

31. Les deux autres occurrences se rencontrent dans l'inscription funéraire mentionnée précédemment (n. 24) et dans un fragment provenant du Thesmophorion (Cl. Rolley, " Le sanctuaire des dieux patrôoi et le Thesmophorion de Thasos ", BCH 89 [1965], p. 452, no 12, ca 480). 
La forme des lettres, si on la compare aux inscriptions lapidaires thasiennes, orienterait vers les documents datés du début du ve s. Mais le caractère privé du texte ainsi que le support peu fréquent rendent difficiles les comparaisons avec l'écriture en usage dans les documents officiels. Ce sont en revanche certains critères internes concernant l'alphabet qui peuvent aider à préciser la date (tabl. I).

\section{2. Concomitance Des AlPhabets PARIEN ET IONIEN ORIENTAL}

Les alphabets archaïques des Cyclades, comme la majorité des alphabets archaïques, ne font pas la distinction dans la valeur de /o/, l'omicron notant toute voyelle de timbre $o$, quelles que soient sa quantité et son aperture. Au sein de ce groupe, l'alphabet parien fait exception puisqu'il distingue les valeurs du /o/ en utilisant les deux lettres, $\Omega$ et $\mathrm{O}$. Mais les valeurs de ces graphèmes sont inversées, marque d'une adaptation locale de l'alphabet milésien : $\Omega$ note en effet /o/ ainsi que /o :/ issu de contraction ou d'allongement compensatoire, tandis que $\mathrm{O}$ note $/ \mathrm{o}: /$, dans un système qui est exactement l'inverse de celui de l'alphabet milésien, et ce de manière tout à fait constante.

Or une difficulté majeure de ce texte réside justement dans la distinction entre $\Omega$ et $\mathrm{O}$, deux lettres rondes dont le tracé est ici approximatif. Il semble bien toutefois que le graveur a cherché à distinguer ses $\Omega$ en en faisant une lettre ronde légèrement ouverte dans sa partie inférieure. Ainsi lit-on nettement 1. 2 un oméga dans la désinence de génitif

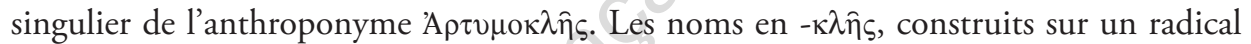
sigmatique ${ }^{32}$, présentent en ionien un génitif en $-\kappa \lambda \varepsilon_{0} \varsigma^{33}$. Par conséquent, la graphie

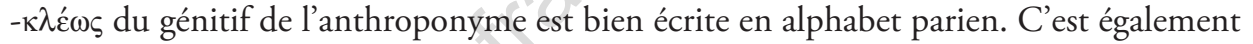
ce qui ressort de la graphie de l'anthroponyme de la l. 1, si l'on admet la lecture du

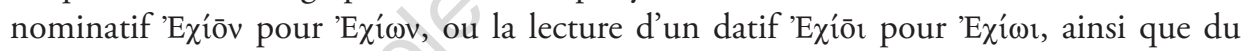
composé en - $\alpha \rho \chi \circ \varsigma 1.3$, écrit- $\alpha \rho \chi \omega \varsigma$.

L'inversion entre $\mathrm{O}$ et $\Omega$ n'est toutefois plus opératoire à la ligne 4 : en effet le groupe

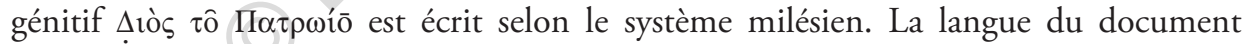
présente par conséquent de manière concomitante les deux systèmes de notation pour la valeur $\mathrm{du} / \mathrm{o} /$, dans un alphabet transitoire mélangeant des graphies pariennes et ioniennes orientales. Cela semble indiquer qu'il existe en outre un critère de répartition entre les deux systèmes de graphies : la graphie parienne, locale, est privilégiée dans les anthroponymes, peut-être plus sujets à une volonté de conservatisme, tandis que la graphie ionienne qui va se généraliser est quant à elle utilisée dans le lexique. Demeure toutefois rétif à l'interprétation le mot de la 1. 5.

32. Le radical est $-\kappa \lambda \dot{\varepsilon}(F) \varepsilon \sigma-$.

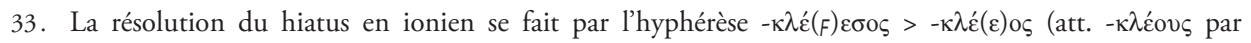
contraction), Fr. Bechtel, Die griechischen Dialekte III. Der ionische Dialekt (1884) $\$ 17.1$ et $\$ 137.2$. 


\section{3. Datation}

La stèle funéraire de Philis que les critères paléographiques datent des alentours de 430$425^{34}$ constitue un jalon important dans l'évolution des graphies des inscriptions thasiennes puisqu'il s'agit de la première inscription où l'alphabet parien est totalement absent. Le reste de la documentation atteste encore un alphabet transitoire jusque vers 420 , date à partir de laquelle les textes sont rédigés dans l'écriture ionienne ${ }^{35}$. Au vu des phénomènes observés dans l'épigraphie lapidaire, la présence dans ce document de graphies à la fois en alphabet parien et en alphabet ionien oriental invite donc à le dater aux alentours de 450-430. Cette datation, qui se fonde sur une comparaison avec l'épigraphie lapidaire, ne peut cependant être qu'indicative puisque, selon toute vraisemblance, l'adoption de nouvelles graphies dans l'épigraphie privée ne se fait pas suivant le même rythme que dans l'épigraphie publique. Aussi n'est-il pas impossible, en raison du caractère " ancien " de la forme de certaines lettres, que cette date puisse être remontée de quelques dizaines d'années, au deuxième quart du ve s. ${ }^{36}$.

\section{COMMENTAIRE}

Avant d'aborder le commentaire du contenu lui-même, il convient de procéder à quelques remarques linéaires.

L. 1 : l'anthroponyme 'Exí $\omega v$ n'est pas attesté pour le moment dans l'épigraphie grecque, excepté à Pompéi et en Occident, autour du $\mathrm{I}^{\mathrm{er}} \mathrm{s}$. apr. J.-C. ${ }^{37}$. Est attesté cependant l'anthroponyme 'Exías, construit sur le même radical mais raccourci différemment ${ }^{38}$. On a noté la difficulté de la lecture du $\mathrm{N}$ final dont on ne voit qu'une haste verticale. On ne peut par conséquent totalement exclure qu'il s'agisse d'un I : 'Exíôı, auquel cas nous aurions à faire au datif d'un nom 'Exıs. Ce nom n'est pour le moment pas attesté

34. IG XII 8, 401 (Pouilloux 1954, pl. X) mais B. Holtzmann fait remonter le relief à 450-440 (B. Holtzmann, Étude de la sculpture thasienne. Les reliefs [1988], p. 661-671).

35. L'évolution de la langue est particulièrement visible dans les bornes des patrai retrouvées au Thesmophorion car elles forment un groupe homogène et pertinent pour l'étude de l'alphabet transitoire.

36. Si la diffusion de l'alphabet ionien oriental en Macédoine et en Chalcidique a pu être favorisée dans les toutes premières années du ve s. par l'avancée vers l'Ouest de l'armée perse qui comportait des Ioniens (A. Panayotou, "Dialectal Inscriptions from Chalcidice, Macedonia and Amphipolis ", dans Third International Symposium on Macedonia [1996], p. 124-163), rien ne permet de l'affirmer pour le corpus thasien.

37. A Lexicon of Greek Personal Names (1987), www.lgpn.ox.ac.uk, tome IIIA. On le rencontre toutefois dans les sources littéraires, comme le nom d'un participant à l'expédition des Argonautes.

38. Fr. Bechtel, Die historischen Personennamen des Griechischen bis zur Kaiserzeit (1917), p. 183 (Ive s. av. J.-C.). 
à la différence d'Exías, mais il pourrait s'agir d'un hypocoristique formé sur un premier élément de composé en -i- du type 'A $\lambda \varepsilon \xi \xi^{\prime}-v i \kappa o s$, d'où $A \lambda \varepsilon_{\varepsilon} \xi_{10}{ }^{39}$.

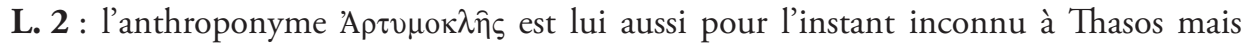
de nombreux noms composés avec Aptv- au premier membre y sont bien attestés, notamment dans le catalogue des théores (entre le $\mathrm{VI}^{\mathrm{e}}$ et le $\mathrm{III}^{\mathrm{e}} \mathrm{s}$.) : on rencontre ainsi

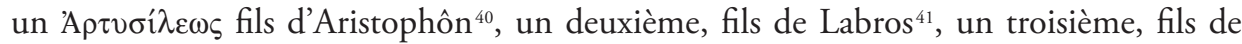
Lallès ${ }^{42}$, un quatrième, fils de Pythiôn ${ }^{43}$, et un autre, père de Dôrothéos ${ }^{44}$. Ailleurs dans

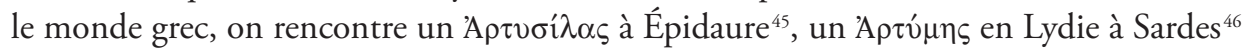
- dont $\mathrm{O}$. Masson émettait l'hypothèse qu'il pouvait s'agir d'un nom lydien, variante

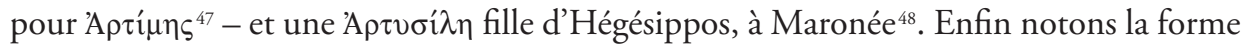
raccourcie Aprv́ $\lambda$ oos à Thèbes en Béotie ${ }^{49}$.

Le terme öpruৎ est connu par deux gloses d'Hésychius ${ }^{50}$ :

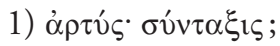

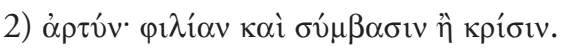

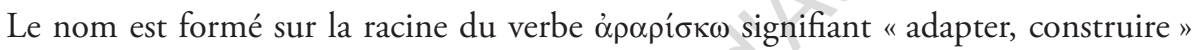
ou "être adapté, convenir» pour les formes intransitives. Selon P. Chantraine, le mot

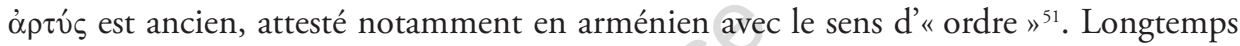
connu par le seul témoignage d'Hésychius, le terme est cependant attesté dans un décret de Thasos pour trois juges de Cos, datant du milieu ou de la seconde moitié du $\operatorname{III}^{\mathrm{e}} \mathrm{s}^{52}$ :

39. Ce parallèle m’a été suggéré par S. Minon.

40. IG XII Suppl 370, 1. 4. Ce terme se rencontre également sous la forme d'un appellatif pour désigner un ministre des sacrifices à Délos chez Athénée (Ath., Deipnosophistes 173a. Dans ce même passage est également attesté un ò $\rho \tau v \sigma i ́ \tau \rho \alpha \gamma o \varsigma)$.

41. Pouilloux 1954, cat. 1, col. 5, 1. 52.

42. Ibid., cat. 1, col. 2, 1. 27.

43. Ibid., cat. 1, col. 4, 1. 54.

44. Ibid., cat. 1, col. 4, 1. 56 .

45. IG IV $\mathrm{IV}^{2} 1,94 \mathrm{~b}, 1.31$; seconde moitié du IVe s. av. J.-C.

46. $S E G 36,1011,20,22,31,39$; IV s. av. J.-C.

47. O. Masson, «L'inscription d'Éphèse relative aux condamnés à mort de Sardes (IEphesos 2) ", REG 100 (1987), p. 225-239.

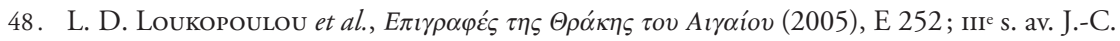

49. SEG 28 466, 2; 338 av. J.-C.

50. Hes. s. $-v$.

51. P. Chantraine, Dictionnaire étymologique de la langue grecque ${ }^{2}$ (2009).

52. Ch. V. Crowther, "Aus der Arbeit der "Inscriptiones Graecae" IV. Koan Decrees for Foreign Judges ", Chiron 29 (1999), p. 251-319, n 3, 1. 13-15 (K. J. Rigsby, IG XII 4, 136; P. Hamon, Documents publics du IVe siècle et de l'époque hellénistique, inédit [à paraître], n²1). 


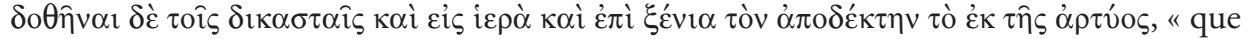
l'apodecte verse aux juges, à la fois pour les sacrifices et comme présent d'hospitalité, la somme prévue par la loi »(trad. P. Hamon).

À la fin de leur mission, ces juges de Cos venus régler des litiges à Thasos, se voient octroyer un certain nombre d'honneurs. Ph. Gauthier interprétait l'expression " $\dot{\kappa} \kappa \hat{\eta} \varsigma$

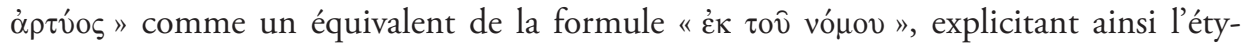

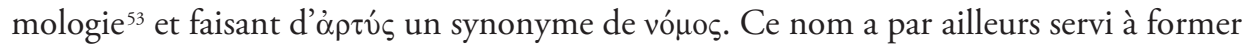
les verbes $\alpha \rho \tau v ́ \omega$ et $\alpha \rho \tau v ́ v \omega$, dont le sens est " arranger, préparer, ajuster/assaisonner ».

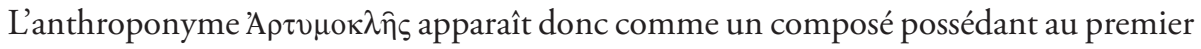
membre un dérivé de $\alpha \rho \tau u ́ \omega$ avec élargissement au moyen du suffixe - $\mathrm{m}(\mathrm{o})^{54}$ et au second

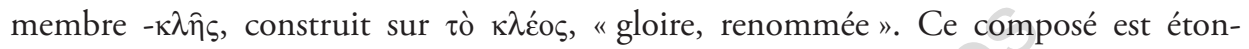
nant du point de vue morphologique : on attend en effet au premier membre la forme $\dot{\alpha} \rho \tau v-$ ou $\alpha \rho \tau v \sigma 1-$, comme en témoignent les exemples cités précédemment ${ }^{55}$. Il faut par conséquent analyser ce composé comme une formation secondaire qui ne répond pas au modèle attendu mais qui est analogique : à partir d'un hypocoristique **'A $\rho \tau v-\mu$-o pour

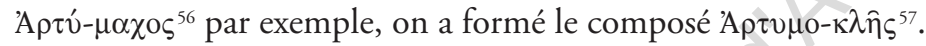

Cet hapax, dont le second membre est courant à Thasos dans l'anthroponymie, augmente ainsi le fonds de noms composés portés par l'élite thasienne aux époques archaïque et classique.

L. 3 : en nous fondant sur ce qu'il reste des premières lettres et sur le nombre de lettres à restituer à gauche, nous proposons la lecture [E] v้ $\alpha \rho \chi o \varsigma$. La consultation du LGPN montre que cet anthroponyme est bien attesté dans tout le monde grec (entre autres exemples à Chypre, à Délos, en Eubée, à Rhodes, en Étolie, en Argolide, en Arcadie...) et se rencontre aussi sous des formes dérivées comme Euarchidès/Euarchidas. Comme pour les deux anthroponymes précédents, notre document présente la première attestation de ce nom dans l'épigraphie thasienne.

53. BullÉp 2000, 502.

54. Dont on trouve un exemple sous la forme du substantif neutre en - $\mu \alpha$ chez Hésychius : $\alpha^{\prime} \rho \tau v \mu \alpha \cdot \delta 1 \alpha \theta \eta \dot{\kappa} \kappa$.

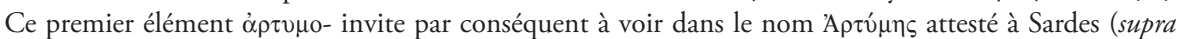
n. 46) non pas un nom lydien, comme le pensait O. Masson (O. Masson [n. 47]), mais plutôt un nom construit sur un radical d'origine grecque.

55. Voir également Fr. Bechtel (n. 38), p. 77-78.

56. On attendrait également ${ }^{* *} A \rho \tau v^{\prime}-\mu-\alpha \varsigma$ ou **'A ${ }^{*} \tau \dot{-}-\mu-\omega v$.

57. Cette hypothèse est due à $S$. Minon à qui je renouvelle mes remerciements. 
Les marques de ponctuation, que l'on rencontre à Thasos encore au milieu du IVe $s$. comme l'indique la Stèle des Braves ${ }^{58}$, apparaissent sous la forme des trois points et séparent nettement l'en-tête de ce qui suit ${ }^{59}$.

L. 4 sq. : s'il est possible de déchiffrer le groupe au génitif de la ligne 4, l'établissement des lignes suivantes pose de réelles difficultés. Dans une lettre adressée à O. Picard au sujet de ce document ${ }^{60}$, J. Pouilloux proposait pour la ligne 5 la restitution [-]Ẹ غ̇ต́pos ФAI. Si l'on reconnaît la deuxième personne de l'imparfait de ópó- $\omega$, en revanche la séquence $\Phi A I$ n'est pas satisfaisante, aucun $\Phi$ n'apparaissant sur la plaque. On aurait par conséquent la restitution éópas AI, qui ne convient pas non plus complètement puisque, dans la partie conservée du document, les lignes respectent la coupe des mots.

Une autre proposition serait de lire éćp $\alpha \sigma \alpha l^{61}$, parfait moyen passif à la deuxième personne du singulier, mais le sens n'apparait pas clairement et la séquence des lettres qui précèdent, si c'est bien [-]ET, rend difficile cette hypothèse ${ }^{62}$. Une autre hypothèse

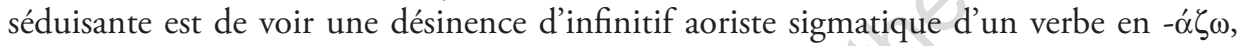

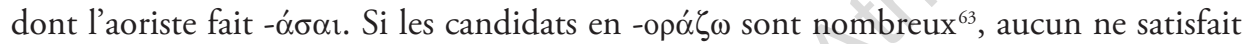
avec le E qui précède et dont la lecture ne fait quasiment pas de doute.

Les restitutions que nous proposons enfin pour les lignes suivantes sont très hypothétiques. À la 1. 6, il est tentant de lire la terminaison d'un imparfait à la troisième personne du singulier, suivi du pronom personnel à la deuxième personne, ouvrant peut-

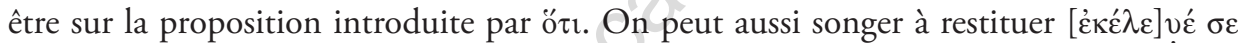
suivi d'un relatif indéfini du type ó $\tau$ เov̂v, dont la gravure s'étendrait sur les lignes 6 et 7 , ce qui n'est pas non plus totalement satisfaisant. Enfin, 1.8 se lit peut-être une désinence de datif, précédant la conjonction öv.

58. Pouilloux 1954, no 141 et J. Fournier, P. Hamon, «Les orphelins de guerre de Thasos: un nouveau fragment de la Stèle des Braves (ca 360-350 av. J.-C.) », BCH 131 (2007), p. 309-381, complétée par un troisième fragment, P. HamoN, "Études d'épigraphie thasienne III. Un troisième fragment de la Stèle des Braves et le rôle des polémarques à Thasos ", $B C H 134$ (2010), p. 301-315.

59. Voir infra.

60. Cette lettre est aujourd'hui conservée aux archives de l'EFA avec les carnets de la fouille.

61. Le dialecte est psilotique.

62. La lettre dans l'épaufrure pose de réels problèmes de lecture et empêche la compréhension de cette ligne. Voir notes critiques.

63. Voir P. Kretschmer, E. Locker, Rückläufiges Wörterbuch der griechischen Sprache ${ }^{2}$ (1963). Par ailleurs, selon l'éclairage, on peut avoir l'impression de distinguer un $p i$ à la place du rhô, ce qui inviterait à lire l'infinitif aoriste de ỏ $\pi \alpha ́ \zeta \omega$ (donner, procurer) mais l'examen attentif de la tablette confirme qu'il s'agit bien d'un $p i$. 


\section{1. UNE LETTRE}

Bien que l'établissement du texte soit difficile, plusieurs indices nous invitent à l'interpréter comme une lettre adressée par un certain Euarchos à Echiôn/Echios fils d'Artumoklès, ainsi que l'atteste la formule introductive. L'auteur de la lettre s'exprime ici à la troisième personne, comme l'indiquent le nominatif Euarchos et le verbe $\kappa \varepsilon \lambda \varepsilon v ́ \varepsilon$, et utilise la deuxième personne pour s'adresser au destinataire, exprimé par l'accusatif du pronom $\sigma \varepsilon$ à la $1.3^{64}$. La principale difficulté réside par conséquent dans la lecture que l'on fait de la désinence du nom à la 1.1 : la lecture 'Exíōv implique un nominatif65. Le genre épistolaire invite le plus souvent à exprimer le nom du destinataire au datif mais les exemples où la formule introductive s'apparente à une adresse avec ce nom au vocatif ne sont pas rares ${ }^{66}$. La lecture 'Exíôı lève quant à elle de manière définitive la difficulté puisque le nom du destinataire serait comme attendu au datif, ce qui semble une solution plus satisfaisante.

Un deuxième indice est la façon dont la formule introductive est mise en valeur par une volonté de mise en page : l'examen des deux premières lignes montre clairement qu'il n'y a pas de lettre à gauche. Par ailleurs, les lettres sont légèrement plus grandes que dans le reste du document. S'ajoute enfin la présence du vacat à droite, autant d'éléments qui indiquent une mise en exergue délibérée du nom et du patronyme du destinataire, Echiôn/Echios fils d'Artumoklès. Suit à la ligne 3, malheureusement mutilée à gauche, le nom de l'expéditeur, désigné par son seul idionyme, Euarchos. Ce nom ne bénéficie pas d'une mise en page spéciale mais il est toutefois isolé de la suite du texte, qui constitue le corps du message, par la ponctuation.

C'est en effet à partir de la ponctuation 1.3 qu'est délivré le contenu du message à proprement parler, qui se développe sous la forme d'une requête de l'auteur au destinataire: le verbe $\kappa \varepsilon \lambda \varepsilon v ́ \varepsilon i$ est ici construit avec son régime à l'accusatif $(\sigma \varepsilon)$, de sorte que l'on attend dans la suite du texte un infinitif, "Euarchos te demande de faire

64. On ne peut non plus exclure qu'Euarchos, l'auteur du message, et le scribe sont deux personnes distinctes.

65. Il est en effet avéré qu'il n'y a pas de iota après le nu restitué, voir supra notes critiques. Ce nominatif aurait alors valeur de vocatif, comme dans CeCCARELli 2013, n 7, p. 340. La forme de vocatif attendue

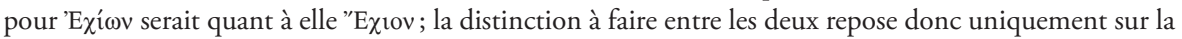
lecture du /o/ et sur la présence ou non de l'alphabet parien.

66. On en trouve ainsi six exemples dans le corpus des lettres du monde grec rassemblé par P. Ceccarelli, la plus ancienne étant une lettre de Bérézan d'un certain Achillodôros à son fils Protagoras et Anaxagoras (seconde moitié du vi ${ }^{\mathrm{e}}$ s.) où, après une adresse au vocatif, Achillodôros parle à son fils en s'exprimant à la troisième personne, comme dans notre document, voir CecCARELli 2013, no 1, p. 335 (Éd. pr. Y. Vinogradov, « Drevnejsee greceskoe pis'mo s ostrova Berezan » [The most Ancient Greek Letter from the Island of Berezan], VDI 4 [1971], p. 74-100). Les autres exemples où le destinataire est au vocatif sont Ceccarelli 2013, no 7, p. 340 ; no 18 , p. 344 ; no 31, p. $350 ; n^{\circ} 35$ et 37, p. 351. P. Ceccarelli a justement montré que, jusqu'au IVe s., le genre épistolaire ne faisait l'objet d'aucune forme codifiée et était régi davantage par des spécificités locales que par des normes stylistiques. 
quelque chose $»^{67}$. La ligne 5 , dont malheureusement la lecture est difficile, pourrait faire apparaître cet infinitif, sous la forme d'un aoriste sigmatique en - $\sigma \alpha$ l.

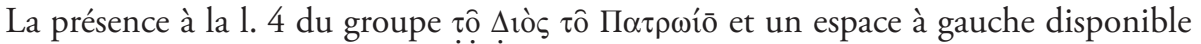
pour trois ou quatre lettres invitent à restituer une préposition régissant le groupe au génitif. Les prépositions à sens géographique pourraient ainsi convenir : $\dot{\alpha} \pi$ ( en

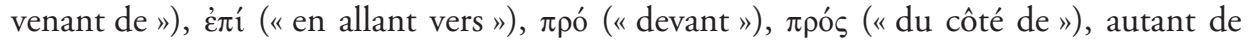

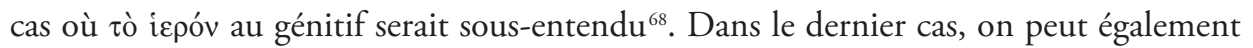

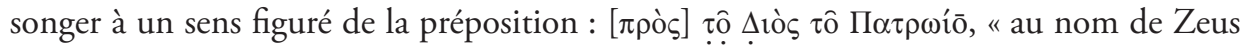
Patrôos ». La préposition $\pi$ pós, suivie de $\theta \varepsilon \hat{\omega} v$ ou d’un nom de divinité, peut en effet se rencontrer dans les formules de supplication ou de serment, garantissant la caution de la divinité dans cet acte d'énonciation ${ }^{69}$. Dans cette hypothèse, Euarchos placerait donc sa

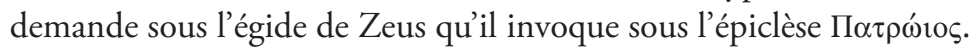

Lépiclèse est très répandue dans le monde grec et indique que la divinité ainsi qualifiée est reconnue comme héritée des ancêtres. L'analyse des documents épigraphiques montre par ailleurs que, dans la réalité du culte, l'épiclèse se trouve au croisement de trois sphères : la sphère de l'individu et de sa famille, celle du groupe civique et, plus généralement, celle de la cité dans son ensemble. Les dieux patrôoi sont en effet honorés par les subdivisions civiques dont le nom et la nature varient d'une cité à l'autre (phratries, tribus ou patrai) mais aussi par la famille ${ }^{70}$. À Thasos, les dieux Patrôoi sont connus par une série d'inscriptions mises au jour lors de la fouille menée en contrebas du théâtre par Cl. Rolley au Thesmophorion, sanctuaire situé au Nord de la cité, à l'extérieur du rempart ${ }^{71}$. L'existence du culte a ainsi été mise en évidence par la découverte de neuf stèles (bornes, autels et règlements religieux) sur une petite terrasse située au pied du mur de soutènement du sanctuaire. Mentionnant le

67. On rencontre le verbe $\kappa \varepsilon \lambda \varepsilon v ́ \omega$ avec cette valeur d'instruction dans une lettre gravée sur plomb provenant de la région de Marseille où l'auteur termine son message par кєке́ $\lambda \varepsilon v \kappa \kappa \alpha$, « telles sont mes instructions ».

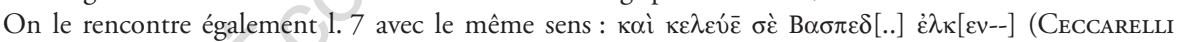
2013, no 23, p. 346).

68. On pourrait pour l'aoriste songer alors à un verbe du type $\pi \varepsilon p \alpha ́(\omega$, mais la présence de l'omicron 1.5 empêche cette restitution. En outre on attendrait, en langue ionienne, $\pi \varepsilon \rho \hat{\sigma} \sigma \alpha$.

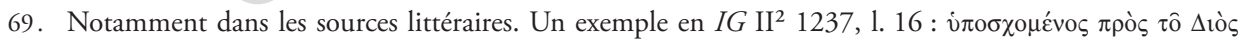

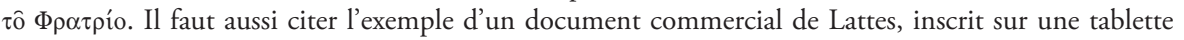
de plomb, où Zeus est invoqué au vocatif précédé de $\hat{\text { S }(M . ~ B A T S, ~ " U n e ~ l e t t r e ~ s u r ~ p l o m b ~ a ̀ ~ L a t t e s ~ ", ~}$ Lattara 21 [2010], p. 749-756).

70. Si cette distinction implique, dans la mesure du possible, de faire la part entre contexte privé et contexte public, dans le cas de Zeus Patrôos il est toutefois souvent difficile de déterminer la frontière entre l'implication civique et domestique du dieu, dans laquelle il s'apparente à un daimôn, au même titre que Zeus

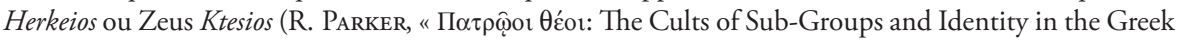
World", dans A. H. et S. W. Rasmussen, Rituals, Resources and Identity in the Ancient Graeco-Roman World, The BOMOS-Conferences 2001-2005 [2008], p. 201-214).

71. Cl. Rolley, "Le sanctuaire des dieux patrôoi et le Thesmophorion de Thasos ", BCH 89 (1965), p. 441-483. 
nom de plusieurs divinités portant chacune l'épiclèse Patrôos, ces bornes marquaient dans le sanctuaire les emplacements réservés à chaque patrè pour célébrer le culte du dieu sous la protection duquel elle se plaçait ${ }^{72}$. Quatre bornes délimitaient ainsi la portion du sanctuaire réservée au Zeus des groupes civiques et familiaux, honoré sous les épiclèses Ktesios et Alastoros $^{73}$. À ces témoignages, il faut en ajouter un cinquième, une borne provenant de l'agora ${ }^{74}$, marquant le temenos de Zeus Ktesios Patrôos.

L'aspect mutilé de la suite du document empêche malheureusement toute interprétation sur le contenu de la requête adressée par Euarchos. Toutefois, l'invocation à Zeus Patrôos, si l'on privilégie la préposition $\pi \rho o ́ \varsigma$ avec un sens figuré, peut peut-être s'expliquer par le contenu privé du message : ayant à régler une affaire domestique, l'auteur invoque tout naturellement le dieu qu'il considère comme le dieu de la patrè à laquelle il appartient ou de son groupe familial. Si l'on restitue au contraire une préposition à sens géographique, on comprendra qu'Euarchos prend, dans sa demande, un sanctuaire de Zeus Patrôos comme indication topographique, peut-être un de ceux situés dans le Thesmophorion, celui de l'agora ou bien un autre plus proche de la maison dans laquelle fut trouvé le document. Malheureusement, le fait que la tablette ait fait l'objet, comme le reste du matériel d'époque archaïque et classique, d'un enfouissement à l'occasion du réaménagement de l'édifice à l'époque hellénistique empêche d'établir un lien précis entre le texte et le contexte archéologique.

Enfin, l'espace laissé libre à gauche à la ligne 7 pose lui aussi question : est-on passé à une autre partie du message que la mise en page souhaitait séparer nettement?

\section{2. LA QUESTION DU SUPPORT}

Les lettres du monde grec qui nous sont parvenues sont inscrites sur des lamelles de plomb, incisées ou peintes sur des tessons après cuisson ou écrites sur papyrus ${ }^{75}$.

72. Sont ainsi attestés Athéna Patrôè, Athéna Mykésiè, les nymphes Patrôai, Artémis Patrôè. Sur ces bornes est gravé le nom du dieu au génitif (dépendant d'un őos ou $\tau \varepsilon ́ \mu \varepsilon v o \varsigma$ sous-entendu), suivi éventuellement du génitif du groupe civique lui rendant le culte. La présence du culte des dieux Patrôoi dans l'enceinte même du Thesmophorion et non pas dans l'espace civique comme on pourrait s'y attendre, ne doit pas étonner selon Cl. Rolley, si l'on songe à l'épisode, illustré par Polygnote, des orgia de Déméter transportés de Paros à Thasos par Cléoboia, faisant de Déméter la déesse essentielle de l'entreprise coloniale.

73. Sont ainsi connues les patrai Anchialideis, Phastadeis, Neophantideis et Péléides (Cl. Rolley [n. 71], $\left.\mathrm{n}^{\mathrm{os}} 1-4\right)$.

74. IG XII Suppl. 407.

75. M. Guarducci, Epigrafia Greca III. Epigrafi di carattere privato (1974), p. 317-322; M. L. LANG, Graffiti and Dipinti. The Athenian Agora 21 (1976), p. 8-11; S. Lewis, News and Society in the Greek Polis (1996), p. 142-152; P. A. Rosenmeyer, Ancient Epistolary Fictions. The Letters in Greek Litterature (2001); Chr. Pébarthe, Cité, démocratie et écriture. Histoire de l'alphabétisation d'Athènes à l'époque classique (2006); CecCarelli 2013. 
Les sources littéraires attestent en outre des tablettes de bois enduites de cire ou blanchies $^{76}$.

Le support de ce document thasien est donc tout à fait original : la lettre a été écrite sur l'argile crue de la tablette au moyen d'un stylet puis a été cuite après séchage. Les bords parfaitement plats de la plaquette attestent l'existence d'un cadrage en bois lors du façonnage et du lissage de l'argile crue. L'absence de marque de calcination indique clairement que la cuisson du matériau a été maîtrisée et que la tablette n'a donc pas été cuite accidentellement dans un incendie. Les parallèles qui viennent d'emblée à l'esprit pour ce type de support d'écriture sont évidemment les tablettes d'argile caractéristiques de l'écriture au Moyen Orient ou les tablettes mycéniennes, cuites dans les incendies des palais. Mais dans le monde grec, ce type de support est tout à fait exceptionnel.

Nous n'avons pu trouver qu'un seul autre exemple d'un document inscrit sur une tablette d'argile. Il s'agit également d'une lettre, malheureusement aujourd'hui perdue, qui n'a pu être étudiée par G. Dunst qu'à partir de photographies et d'un fac-similée ${ }^{77}$. Le document, inscrit sur une plaque d'argile entière ${ }^{78}$, a été trouvé dans la région du golfe de Roses en Espagne, entre La Escala et Ampurias (anc. Emporion). Le texte est écrit boustrophédon, dans le dialecte attique mais adopte certaines graphies épichoriques. G. Dunst a proposé la date de 500 environ. L'auteur de cette lettre est un certain Energos $\mathrm{d}^{\prime}$ " Emphylion ${ }^{79}$, potier de métier, qui écrit à son frère au sujet d'argile et de techniques propres à la fabrication des vases.

Il est étonnant qu'aucun des éditeurs de ce texte n'ait relevé le caractère rarissime chez les Grecs de ce type de support pour l'écriture. Il convient de se demander si dans ce cas précis le choix de la tablette d'argile n'a pas été dicté par le métier de l'auteur du message : étant potier, c'est tout naturellement qu'il a pu se tourner vers ce type de matériau dont il disposait en abondance et qu'il pouvait cuire lui-même au milieu de ses vases.

76. Les tablettes, dans les sources littéraires, sont désignées par le terme $\gamma \rho \alpha \mu \mu \alpha \tau \varepsilon i ̂ o v$, par le diminutif $\gamma \rho \alpha \mu \mu \alpha \tau \varepsilon i ́ \delta$ iov ou encore par $\delta \varepsilon ́ \lambda \tau o \varsigma$. Toutefois ces termes désignent au sens propre ce sur quoi on écrit, un adjectif précisant parfois s'il s'agit de tablettes de bois, de cire ou encore de bronze.

77. G. Dunst, "Ein griechisches Töntäfelchen von der Küste bei Ampurias ", MDAI(M) 10 (1969), p. 146-154 et pl. 17. Voir Ceccarrelli 2013, no 25, p. 347-348 pour les éditions ultérieures. Les conditions particulières dans lesquelles le document est parvenu à G. Dunst (l'inventeur du document n'expédia en effet à G. Dunst que quelques photographies et disparut ensuite) a pu lui faire craindre qu'il se fût agi d'un faux.

78. La tablette est toutefois brisée en cinq fragments. Ses dimensions ne sont malheureusement pas connues.

79. La formule introductive de la lettre ne comporte que l'anthroponyme Energos, au nominatif. S'il s'agit du nom de l'expéditeur, le nom du destinataire serait peut-être inscrit sur une autre tablette avec laquelle elle formerait un diptyque. 
L'aspect exceptionnel d'une tablette d'argile comme support d'écriture apparaît d'autant plus au rappel d'un passage de L. Jeffery en 1961: "The various prepared materials on which Greek inscriptions were written have been often listed already by modern scholars. They are: stone, wood, metal, papyrus, leather, waxed tablets, and (according to the traditions of the Greeks themselves) linden-bark and palm or other leaves. Clay tablets were not used; the reason for this neglect of a cheap and abundant medium is not known, but may perhaps have been due at least in part to the force of convention, since the Phoenicians, the Greeks' first teachers of writing, did not use them either. ${ }^{80} "$

Il est en effet assuré que l'absence presque totale de ce type de matériau dans les fouilles indique qu'il n'était guère utilisé pour l'écriture. À la raison avancée par Jeffery, qui paraît peu satisfaisante, on peut ajouter l'obstacle que peut représenter la phase intermédiaire que ce matériau implique : écrire sur une tablette d'argile demande en effet d'aller la porter dans un four de potier afin qu'il la cuise, étape qui n'est pas nécessaire si l'on grave son message sur un tesson de céramique ou sur une lamelle de plomb. Les exemples de Thasos et d'Emporion nous montrent cependant que ce type de support n'est pas à négliger et qu'il a très bien pu être privilégié à l'occasion, notamment s'il constituait le matériau le plus commode pour l'auteur en raison de sa facilité d'utilisation (comme c'est le cas pour Energos) ou de sa pérennité ${ }^{81}$.

Malgré les nombreuses difficultés rencontrées pour déchiffrer ce texte, nous proposons pour les premières lignes la traduction suivante :

"Ô Echiôn vel à Echios fils d'Artymoklès, Euarchos; il te demande, au nom de Zeus Patrôos vel du côté du sanctuaire de Zeus Patrôos ${ }^{82}$, de [-] ".

Cette formule introductive nous assure qu'il s'agit d'une lettre, dont malheureusement le contenu reste énigmatique. Nombreuses sont en effet les questions qui demeurent sans réponse : la nature de la demande d'Euarchos ou les raisons qui ont guidé le choix de l'argile comme support de l'écriture. Toutefois ce texte, si mutilé soit-il, recèle en luimême un certain nombre d'éléments qui méritaient d'être soulevés. Du point de vue de l'écriture tout d'abord. Comme souvent à Thasos, les graffites témoignent de graphies qui ne sont pas ou peu attestées dans l'épigraphie lapidaire. C'est le cas ici pour deux formes spécifiques : \ et + . Le document livre ensuite trois nouveaux anthroponymes à Thasos, dont l'un, Artymoklès, est un hapax. Enfin, nous avons une attestation supplémentaire

80. L. H. Jeffery, A. W. Johnston, The Local Scripts of Archaic Greece ${ }^{2}$ (1990), p. 51.

81. Par conséquent, on peut aussi se demander si le terme $\gamma \rho \alpha \mu \mu \alpha \tau \varepsilon \hat{\imath o v} / \gamma \rho \alpha \mu \mu \alpha \tau \varepsilon i ́ \delta$ เov rencontré dans les sources littéraires ne désigne pas, en plus de tablettes de bois ou de cire, des tablettes d'argile.

82. Selon la préposition restituée, on traduira " en venant du sanctuaire ", " en allant vers le sanctuaire " ou encore "devant le sanctuaire». 
du culte de Zeus Patrôos dans la cité. Surtout, ce document fournit un nouvel exemple d'un texte écrit sur une tablette d'argile. Les lignes montantes, qui s'expliquent par la façon dont le scribe tenait le cadre en bois contenant l'argile, la coupe des mots respectée en fin de ligne ainsi que le ductus incertain de quelques lettres pourraient peut-être indiquer que ce message n'a pas été écrit par un scribe professionnel. 